\title{
Theory of Electronic Ferroelectricity
}

\author{
T. Portengen *, Th. Östreich ${ }^{\dagger}$, and L. J. Sham \\ Department of Physics, University of California San Diego, La Jolla, California 92093-0319
}

(28 May 1996)

\begin{abstract}
We present a theory of the linear and nonlinear optical characteristics of the insulating phase of the Falicov-Kimball model within the self-consistent mean-field approximation. The Coulomb attraction between the itinerant $d$-electrons and the localized $f$-holes gives rise to a built-in coherence between the $d$ - and $f$-states, which breaks the inversion symmetry of the underlying crystal, leading to: (1) electronic ferroelectricity, (2) ferroelectric resonance, and (3) a nonvanishing susceptibility for second-harmonic generation. As experimental tests of such a built-in coherence in mixed-valent compounds we propose measurements of the static dielectric constant, the microwave absorption spectrum, and the dynamic second-order susceptibility.
\end{abstract}

\section{INTRODUCTION}

In this paper, we present a theory of ferroelectricity originating from an electronic phase transition, in contrast to the conventional displacive ferroelectricity due to a lattice distortion 11. The electronic ferroelectricity occurs in a strongly correlated electron system, namely, the insulating phase of the Falicov-Kimball model.

The Falicov-Kimball (FK) model was introduced originally [2] as a simple model to explain the metal-insulator transitions observed in certain transition-metal and rare-earth compounds. The model has since been applied extensively to the mixed-valent compounds and heavy-fermion materials. The FK model introduces two types of electrons: itinerant $d$-electrons and localized $f$-electrons. The valence transition is driven by the on-site Coulomb repulsion between the $d$ - and $f$-electrons. A $d$ - $f$ hybridization term may or may not be added to the model. The theoretical solutions for the ground state of the FK model can be divided into two classes. On the one hand, solutions with $f$-occupation as a good quantum number [3, 4 , do not have a built-in coherence between $d$-electrons and $f$-holes. On the other hand, solutions such as the self-consistent mean-field (SCMF) solution [5] and the electronic polaron [6] do have a built-in coherence between $d$-electrons and $f$-holes.

The built-in coherence of the SCMF solution breaks the inversion symmetry of the FK Hamiltonian in the following way. The many-electron eigenstates of the Hamiltonian can be classified into even and odd parity states. The SCMF ground states with even and odd parities are degenerate. A linear combination of the even and odd parity states forms the appropriate ground state for the limit of a vanishing electric field. We shall argue that such degenerate ground states can exist for solutions with $f$-occupation as a good quantum number. Thus, the inversion symmetry breaking is not limited to the SCMF solution.

The primary purpose of this paper [7] is to give a detailed account of the linear and nonlinear optical characteristics of the SCMF solution. The inversion-symmetry-broken ground state possesses the following distinctive properties: (1) electronic ferroelectricity, (2) ferroelectric resonance, and (3) a nonvanishing susceptibility for second-harmonic generation. Solutions of the model without built-in coherence do not have these properties. As experimental tests to distinguish between the two classes of solutions we propose measurements of the static dielectric constant, the microwave absorption spectrum, and the dynamic second-order susceptibility of a mixed-valent compound, for example $\mathrm{SmB}_{6}$.

In recent years, Four-Wave-Mixing (FWM) spectroscopy has emerged as a powerful tool for studying coherence in optically pumped semiconductor systems 8 10]. In a three-beam FWM experiment, two incoming beams of wavevectors $\mathbf{k}_{1}$ and $\mathbf{k}_{2}$ set up a transient polarization grating. A third incoming beam of wavevector $\mathbf{k}_{3}$ diffracts off this grating to produce an outgoing signal in the direction $\mathbf{k}_{4}=\mathbf{k}_{3}+\mathbf{k}_{2}-\mathbf{k}_{1}$. Being a third-order process, FWM is allowed in media with or without inversion symmetry. We pose the question: what happens if the system being probed already has a polarization built into it by nature? An example of such a system is the SCMF solution of the FK model resulting in the Bose-Einstein condensation of $d$ - $f$ excitons.

\footnotetext{
* Present address: Clarendon Laboratory, University of Oxford, Parks Road, Oxford OX1 3PU, United Kingdom

${ }^{\dagger}$ Present address: Institut für Theoretische Physik, Universität Göttingen, Bunsenstrasse 9, D-37073 Göttingen, Germany
} 
As shown below, the built-in polarization leads to a nonlinear optical response to second order in the external field. The mixed-valent system has a nonvanishing susceptibility $\chi^{(2)}(2 \omega, \omega, \omega)$ for second-harmonic generation. The builtin polarization replaces one of the incoming beams of the three-beam FWM experiment. In crystals with inversion symmetry, second-harmonic generation is forbidden in the electric-dipole approximation. In the mixed-valent system the built-in polarization breaks the inversion symmetry, allowing second-harmonic generation to take place. We present a calculation of the second-harmonic susceptibility of a model mixed-valent system within the SCMF approximation. The second-harmonic susceptibility is directly proportional to the built-in coherence $\Delta$, showing that second-harmonic generation can be used as a test of $d-f$ exciton condensation in mixed-valent compounds.

The existence of a built-in polarization in the ground state also means that, according to the SCMF theory, mixedvalent compounds are ferroelectric. Whereas in commonly known ferroelectrics the built-in polarization is due to the relative displacement of positive and negative ions, the ferroelectricity in mixed-valent compounds is of purely electronic origin. Apart from possible Jahn-Teller distortions as a result of the electronic polarization, the valence transition does not involve a change in the crystal structure. As shown below, the valence transition is accompanied by a divergence of the static dielectric constant at the critical value of the $f$-level energy. The divergence of the static dielectric constant should be observable in real mixed-valent compounds, for example by varying the external pressure or temperature.

The continuous symmetry associated with the phase of $\Delta$ leads to a Goldstone mode in the excitation spectrum of the mixed-valent compound. In the pseudo-spin picture, the Goldstone mode corresponds to a uniform precession of the pseudo-spins around the $z$-axis. Ferroelectric resonance occurs when an ac electric field is applied whose frequency coincides with that of the Goldstone mode. This phenomenon is the electric analogue of magnetic resonance in ferromagnetic insulators. The ferroelectric resonance frequency is proportional to the square root of the effective bias field, and may depend on the sample shape, domain structure, and crystal fields. In real mixed-valent compounds, ferroelectric resonance should occur in the microwave regime.

The remainder of the paper is organized as follows. In section [1] we discuss the SCMF solution for the ground state of the FK model. We show that the SCMF ground state has a built-in polarization which breaks the inversion symmetry of the FK Hamiltonian. We calculate the static dielectric constant of a model mixed-valent system using mean-field theory. In section III we calculate the linear susceptibility of the model system using the pseudo-spin formalism. We obtain an analytical expression for the linear susceptibility in a uniform static electric field. We determine the ferroelectric resonance frequency and analyze the shape of the infrared absorption spectrum. In section IV we compute the second-harmonic susceptibility of the model system. In section $\mathrm{V}$ we compare the results of the model calculation to experimental data and propose experimental tests of coherence in real mixed-valent compounds. In section VI we discuss the possibility of ferroelectricity, ferroelectric resonance, and second-harmonic generation for solutions of the FK model other than the SCMF solution. The main results are summarized in section VII.

\section{MODEL}

Ignoring the electron spin, the FK Hamiltonian is

$$
\begin{aligned}
H= & \sum_{\mathbf{k}} \varepsilon_{\mathbf{k}} d_{\mathbf{k}}^{\dagger} d_{\mathbf{k}}+E_{f}^{\prime} \sum_{\mathbf{k}} f_{\mathbf{k}}^{\dagger} f_{\mathbf{k}}+\sum_{\mathbf{k}} V_{\mathbf{k}} d_{\mathbf{k}}^{\dagger} f_{\mathbf{k}}+\text { h.c. } \\
& +\frac{U}{N} \sum_{\mathbf{k}, \mathbf{k}^{\prime}, \mathbf{q}} d_{\mathbf{k}+\mathbf{q}}^{\dagger} d_{\mathbf{k}} f_{\mathbf{k}^{\prime}-\mathbf{q}}^{\dagger} f_{\mathbf{k}^{\prime}} .
\end{aligned}
$$

Here $d_{\mathbf{k}}^{\dagger}$ creates a $d$-electron of momentum $\mathbf{k}$ and energy $\varepsilon_{\mathbf{k}}$, and $f_{\mathbf{k}}^{\dagger}$ creates an $f$-electron of momentum $\mathbf{k}$ and energy $E_{f}^{\prime}$. The parameter $U$ is the Coulomb repulsion between the $d$ - and $f$-electrons, $V_{\mathbf{k}}$ is the hybridization energy, and $N$ is the number of sites. We assume that the $d$-band and the $f$-level are derived from $d$ - and $f$-orbitals on the same site. We have chosen the $d$ - and $f$-orbitals to yield a finite dipole moment between them in the $z$-direction. For simplicity, we consider a model system with a $d$-band of bandwidth $W$ and constant density of states $\rho_{0}=1 /(2 W)$.

\section{A. SCMF solution}

The SCMF solution is analogous to the BCS theory of superconductivity except that the pairing now occurs between a $d$-electron of momentum $\mathbf{k}$ and an $f$-hole of momentum $-\mathbf{k}$ (Ref. [11]). Whereas a Cooper pair carries charge, an electron-hole pair is neutral. The SCMF solution therefore describes an insulator, rather than a superconductor. Pairing between electrons and holes may also occur in a semiconductor placed in an intense coherent laser field [12]. 
In that case, the pairing is externally enforced by the pump field. The mean-field decoupling of the Hamiltonian (1) yields the effective one-particle Hamiltonian

$$
\begin{aligned}
H= & \sum_{\mathbf{k}}\left(\varepsilon_{\mathbf{k}}+U n_{f}\right) d_{\mathbf{k}}^{\dagger} d_{\mathbf{k}}+\left(E_{f}^{\prime}+U n_{d}\right) \sum_{\mathbf{k}} f_{\mathbf{k}}^{\dagger} f_{\mathbf{k}} \\
& +\sum_{\mathbf{k}}\left(V_{\mathbf{k}}-\Delta\right) d_{\mathbf{k}}^{\dagger} f_{\mathbf{k}}+\text { h.c. }-N U n_{f} n_{d}+\frac{N}{U}|\Delta|^{2},
\end{aligned}
$$

where $\Delta=\frac{U}{N} \sum_{\mathbf{k}}\left\langle\psi\left|f_{\mathbf{k}}^{\dagger} d_{\mathbf{k}}\right| \psi\right\rangle$ is the gap parameter, and $n_{d}=\frac{1}{N} \sum_{\mathbf{k}}\left\langle\psi\left|d_{\mathbf{k}}^{\dagger} d_{\mathbf{k}}\right| \psi\right\rangle$ and $n_{f}=\frac{1}{N} \sum_{\mathbf{k}}\left\langle\psi\left|f_{\mathbf{k}}^{\dagger} f_{\mathbf{k}}\right| \psi\right\rangle=1-n_{d}$ are the $d$-band and $f$-level occupancies respectively. The SCMF ground state is $|\psi\rangle=\prod_{\mathbf{k}}\left(u_{\mathbf{k}}+v_{\mathbf{k}} d_{\mathbf{k}}^{\dagger} f_{\mathbf{k}}\right)|0\rangle$, where $|0\rangle$ is the state with no $f$-holes (the normal state), and $u_{\mathbf{k}}=\cos \frac{1}{2} \theta_{\mathbf{k}}\left(v_{\mathbf{k}}=\sin \frac{1}{2} \theta_{\mathbf{k}}\right)$ is the probability amplitude for the pair state $(\mathbf{k},-\mathbf{k})$ to be occupied (unoccupied). The gap parameter $\Delta$ and the $f$-level occupancy $n_{f}$ must be determined self-consistently from

$$
\begin{aligned}
\Delta & =\frac{U}{N} \sum_{\mathbf{k}} \frac{\Delta-V_{\mathbf{k}}}{2 E_{\mathbf{k}}}, \\
n_{f} & =\frac{1}{2 N} \sum_{\mathbf{k}}\left(1+\frac{\xi_{\mathbf{k}}}{E_{\mathbf{k}}}\right) .
\end{aligned}
$$

Here $E_{\mathbf{k}}=\sqrt{\xi_{\mathbf{k}}^{2}+\left|\Delta-V_{\mathbf{k}}\right|^{2}}$ is the quasiparticle excitation energy, with $\xi_{\mathbf{k}}=\frac{1}{2}\left(\varepsilon_{\mathbf{k}}-E_{f}\right)$, where $E_{f}=E_{f}^{\prime}+U n$ is the renormalized $f$-level energy, and $n=n_{d}-n_{f}$ is the inversion. Since neither $E_{f}$ nor $E_{f}^{\prime}$ are known from first principles, we shall treat $E_{f}$ as the materials parameter. Eqs. (3) and (4) are Eqs. (11) and (10) of Ref. [5] at temperature $T=0$, with a k-dependent hybridization. If the crystal has inversion symmetry, the hybridization must satisfy $V_{-\mathbf{k}}=-V_{\mathbf{k}}$. The assumption of a k-independent hybridization in, among others, Ref. [5], is therefore incorrect. If, instead, we assume nearest-neighbour hybridization, we find that $V_{\mathbf{k}}$ is odd in $\mathbf{k}$ and purely imaginary. As can be seen from Eq. (3), the imaginary part of $\Delta$ then vanishes due to the cancellation of terms with $\pm \mathbf{k}$. The real part of $\Delta$ is given by the BCS gap equation

$$
\Delta=\frac{U}{N} \sum_{\mathbf{k}} \frac{\Delta}{2 E_{\mathbf{k}}}
$$

where $E_{\mathbf{k}}=\sqrt{\xi_{\mathbf{k}}^{2}+\left|V_{\mathbf{k}}\right|^{2}+\Delta^{2}}$. Calculation shows that a sufficiently strong hybridization can destroy the gap. In the following we consider the limit where $V_{\mathbf{k}}$ is negligible compared to $U$.

The solution of Eqs. (4) and (5) for our model system is shown in Fig. 1. The figure shows the gap parameter $\Delta$ and the $f$-level occupancy $n_{f}$ as a function of the $f$-level energy $E_{f}$. The gap parameter $\Delta$ is the order parameter of the valence transition. When the $f$-level is far below the bottom of the $d$-band, the system is in the normal state with no $f$-holes, and $\Delta=0$. As the $f$-level is moved upward past the critical value $E_{f}=-E_{c}$, where $E_{c}=W \operatorname{coth}(W / U)$ (in a real material this is achieved by applying pressure or by alloying), $\Delta$ becomes nonzero and the system undergoes a valence transition. In the mixed-valent state, the $f$-level occupancy $n_{f}$ lies between 0 and 1 . The gap parameter reaches the maximum value $\Delta(0)=W /[2 \sinh (W / U)]$ when the $f$-level lies at the center of the $d$-band $\left(E_{f}=0\right)$. This is the half-filling point $n_{f}(0)=1 / 2$. For still higher $E_{f}$, the $f$-level gradually empties out into the $d$-band. The solutions transform according to $\Delta\left(E_{f}\right)=\Delta\left(-E_{f}\right)$ and $n_{f}\left(E_{f}\right)=1-n\left(-E_{f}\right)$. At $E_{f}=E_{c}$, the system returns to a normal state with no $f$-electrons. Since $n_{f}\left(E_{f}\right)$ has no discontinuities, the valence transition is continuous for all values of the Coulomb repulsion $U$.

\section{B. Inversion symmetry breaking}

The key feature of the SCMF ground state $|\psi\rangle$ is that it breaks the inversion symmetry of the FK Hamiltonian. In general, symmetry breaking occurs when the ground state has a lower symmetry than the Hamiltonian. As a well-known example in another area, the Ising model $H_{\text {Ising }}=-J \sum_{<i j>} S_{i}^{z} S_{j}^{z}$ is invariant under $S_{i}^{z} \rightarrow-S_{i}^{z}$, all $i$. Yet the ground state is either one of the broken-symmetry states with built-in magnetization $\pm M_{z}$. The sign of the magnetization is selected by applying an infinitesimal bias field $H_{z}$, and then choosing the lower-energy state.

In the case of the SCMF solution of the FK model, the inversion symmetry in a $d$ - $f$ site is spontaneously broken due to the pairing of $d$-states of even parity with $f$-states of odd parity. Applying the inversion $\hat{J}$ to $|\psi\rangle$ gives the state 


$$
\hat{J}|\psi\rangle=\prod_{\mathbf{k}}\left(-u_{\mathbf{k}}^{*}+v_{\mathbf{k}}^{*} d_{\mathbf{k}}^{\dagger} f_{\mathbf{k}}\right)|\psi\rangle,
$$

which is linearly independent of $|\psi\rangle$. The form of the inversion image follows from the even parity of the $d$-orbital and the odd parity of the $f$-orbital and $u_{-\mathbf{k}}=u_{\mathbf{k}}^{*}$ and $v_{-\mathbf{k}}=v_{\mathbf{k}}^{*}$. The states $|\psi\rangle$ and $\hat{J}|\psi\rangle$ have built-in polarizations $\langle\psi|\hat{\mathbf{P}}| \psi\rangle=\mathbf{P}^{(0)}$ and $\langle\psi|\hat{J} \hat{\mathbf{P}} \hat{J}| \psi\rangle=-\mathbf{P}^{(0)}$, where

$$
\hat{\mathbf{P}}=\frac{\mu}{\Omega} \sum_{\mathbf{k}} d_{\mathbf{k}}^{\dagger} f_{\mathbf{k}}+\text { h.c. }
$$

is the polarization operator. $\boldsymbol{\mu}$ is the interband dipole matrix element, which for simplicity we take to be independent of $\mathbf{k}$, and $\Omega$ is the volume. One can show that in the thermodynamic limit $N \rightarrow \infty,|\psi\rangle$ and $\hat{J}|\psi\rangle$ are orthogonal and uncoupled by $H$. The proof relies on the fact that the infinite product $\prod_{\mathbf{k}}\left(\left|v_{\mathbf{k}}\right|^{2}-\left|u_{\mathbf{k}}\right|^{2}\right)$ is zero, since $\left|\left(\left|v_{\mathbf{k}}\right|^{2}-\left|u_{\mathbf{k}}\right|^{2}\right)\right|<1$ for almost all $\mathbf{k}$.

Because $H$ is invariant under inversion, $|\psi\rangle$ and $\hat{J}|\psi\rangle$ are degenerate. As in the case of the Ising model, the correct ground state is selected by lifting the degeneracy with a bias field $\mathbf{E}$, and then choosing the lower-energy state. This yields the state $|\psi\rangle$ with built-in polarization in the direction of $\mathbf{E}$. We call this direction the $z$-direction. (Without crystal-field terms, the $z$-direction has no definite orientation with respect to the crystal axes.) Since $\mu_{z}$ is real, $P_{z}^{(0)}=N \mu_{z}\left(\Delta+\Delta^{*}\right) /(\Omega U)$, where $\Delta$ is the built-in coherence. The built-in polarization vanishes in the normal state where $\Delta=0$.

\section{Electronic Ferroelectricity}

In commonly known ferroelectrics such as $\mathrm{BaTiO}_{3}$, the ferroelectric transition involves a change in the crystal structure. In the ferroelectric phase, the positive ions are displaced relative to the negative ions, leading to a permanent electric dipole moment. The displacive ferroelectric transition occurs when the transverse optical (TO) phonon frequency vanishes at some point in the Brillouin zone. In an electronic ferroelectric, the ferroelectric transition involves a change in the electronic structure rather than the crystal structure. (Here we neglect the electron-phonon coupling, which may cause a lattice distortion as a secondary effect of the transition.) Instead of a vanishing of the TO phonon frequency, the $d$ - $f$ exciton energy goes to zero at the critical value of the $f$-level energy. The built-in polarization of an electronic ferroelectric is of the order of $10 \mu \mathrm{C} / \mathrm{cm}^{2}$, comparable to the built-in polarization of perovskites [1].

Since the built-in polarization is continuous at $E_{f}= \pm E_{c}$ (see inset of Fig. 2), the valence transition is a secondorder ferroelectric transition. In general, second-order ferroelectric transitions are accompanied by a divergence of the static dielectric constant in the direction of the spontaneous polarization. For a temperature-driven transition, the dielectric constant diverges as $\left(T-T_{c}\right)^{-\gamma}$ above $T_{c}$, and as $\left(T_{c}-T\right)^{-\gamma^{\prime}}$ below. Almost all known ferroelectrics have $\gamma=1$ (Curie-Weiss law). Observed values of $\gamma^{\prime}$ range from $1 / 2$ in TGS to $1 / 6$ in SbSI [13].

Here we calculate the static dielectric constant of the model mixed-valent system at temperature $T=0$, using mean-field theory. The dielectric constant in the $z$-direction is given by $\epsilon_{z z}=1+4 \pi \chi_{z z}^{(1)}$, where $\chi_{z z}^{(1)}=$ $\lim _{E_{z} \rightarrow 0} \partial P_{z}^{(0)} / \partial E_{z}$ is the static susceptibility. For $\Delta$ real, the polarization is $P_{z}^{(0)}=2 N \mu_{z} \Delta /(\Omega U)$, so that $\chi_{z z}^{(1)}=\left[2 N \mu_{z} /(\Omega U)\right] \lim _{E_{z} \rightarrow 0} \partial \Delta / \partial E_{z}$. The bias field $E_{z}$ leads to an additional term $-\mu_{z} E_{z} \sum_{\mathbf{k}} d_{\mathbf{k}}^{\dagger} f_{\mathbf{k}}+$ h.c. in the effective one-particle Hamiltonian. The self-consistency equation for $\Delta$ then becomes

$$
\Delta=\frac{U}{N} \sum_{\mathbf{k}} \frac{\Delta+\mu_{z} E_{z}}{2 E_{\mathbf{k}}}
$$

where $E_{\mathbf{k}}=\sqrt{\xi_{\mathbf{k}}^{2}+\left(\Delta+\mu_{z} E_{z}\right)^{2}}$. The susceptibility is obtained by implicit differentiation of Eqs. (价) and (8) with respect to $E_{z}$. The susceptibility of the normal state $(\Delta=0)$ is

$$
\chi_{z z}^{(1)}=-\frac{2 N \mu_{z}^{2}}{\Omega} \frac{\operatorname{arccoth}\left(\left|E_{f}\right| / W\right)}{W-U \operatorname{arccoth}\left(\left|E_{f}\right| / W\right)},
$$

and the susceptibility of the mixed-valent state $(\Delta>0)$ is

$$
\chi_{z z}^{(1)}=-\frac{2 N \mu_{z}^{2}}{\Omega U}\left(1+\frac{1+4 \Delta^{2} A(0)}{4 \Delta^{2} A(0)\left[1+4 \Delta^{2} A(0)\right]+4 \Delta^{2} B^{2}(0)}\right) .
$$


Here $A(0)$ and $B(0)$ are given by Eqs. (52) and (53) of the Appendix. Fig. 2 shows the dielectric constant of the model system as a function of the $f$-level energy. The dielectric constant diverges at $\left|E_{f}\right|=E_{c}$. From Eq. (9) we find $\chi_{z z}^{(1)} \propto\left(\left|E_{f}\right|-E_{c}\right)^{-1}$ as $\left|E_{f}\right|$ approaches $E_{c}$ from above, and from Eq. (10) we find $\chi_{z z}^{(1)} \propto\left(E_{c}-\left|E_{f}\right|\right)^{-1}$ as $\left|E_{f}\right|$ approaches $E_{c}$ from below. Thus, the critical exponents according to mean-field theory are $\gamma=\gamma^{\prime}=1$.

\section{LINEAR OPTICAL RESPONSE}

We first consider the linear optical response of the mixed-valent system. The SCMF solution predicts an energy gap $2 \Delta$ in the absorption spectrum. The gap is $2 \Delta$ because the incoming photon must create two quasiparticles, just as in a superconductor. Far-infrared transmission and reflectivity spectra [14 16], as well as electron tunneling spectra [17], show energy gaps of several meV in a number of mixed-valent compounds. The crucial difference between the mixedvalent compound and the superconductor is this: in the superconductor, the pairing occurs between two electrons, whereas in the mixed-valent compound the pairing occurs between an electron and a hole. This has important consequences for the coherence factors that enter the response of both systems to different external probes. For example, it is a textbook result [18] that a clean superconductor at temperature $T=0$ cannot absorb electromagnetic radiation because the coherence factor $u_{\mathbf{p}} v_{\mathbf{p}+\mathbf{q}}-v_{\mathbf{p}} u_{\mathbf{p}+\mathbf{q}}$ vanishes for zero photon momentum $\mathbf{q}$. For the mixed-valent compound the coherence factor entering the electromagnetic absorption is $u_{\mathbf{p}} u_{\mathbf{p}+\mathbf{q}}-v_{\mathbf{p}} v_{\mathbf{p}+\mathbf{q}}$, which remains finite for zero photon momentum. The coherence factor entering the electromagnetic absorption of the mixed-valent compound is the same as the coherence factor entering the acoustic attenuation rate of the superconductor [19].

We calculate the linear response of the mixed-valent system to an ac electromagnetic field in the presence of a dc bias field. The bias field serves to select the direction of the built-in polarization. In a real material, the bias field is provided by the crystal field or the depolarization field due to the sample boundary. We treat the interaction of the mixed-valent system with the ac electromagnetic field in the electric-dipole approximation. The interaction term in the Hamiltonian is $H_{\mathrm{int}}=-\mu_{z} \mathcal{E}_{z} \sum_{\mathbf{k}} d_{\mathbf{k}}^{\dagger} f_{\mathbf{k}}+$ h.c., where $\mathcal{E}_{z}$ is the component of the ac electric field along the $z$-direction (i.e. the direction of the built-in polarization). Only the $z$-component of the ac electric field couples to the channel in which the pairing takes place. The optical signatures of $d-f$ exciton condensation occur only in this channel. We ignore the response of the remaining optical channels.

The ac electric field sets up a polarization $P_{z}$ in the material, which in general can be a complicated nonlinear function of $\mathcal{E}_{z}$. In linear response, we expand $P_{z}$ in powers of $\mathcal{E}_{z}$ and keep only the first-order term: $P_{z}^{(1)}=\chi_{z z}^{(1)} \mathcal{E}_{z}$. In the electric-dipole approximation, the linear susceptibility $\chi_{z z}^{(1)}$ depends on the photon frequency $\omega$ but not on the photon momentum q. The quantity measured in experiments is the reflectivity spectrum or the transmission spectrum. From these one can extract the optical conductivity $\sigma_{z z}(\omega)$ by Kramers-Kronig analysis. The optical conductivity is related to the linear susceptibility by $\sigma_{z z}(\omega)=-i \omega \chi_{z z}^{(1)}(\omega)$.

\section{A. Optical Bloch equations}

We have calculated the linear susceptibility $\chi_{z z}^{(1)}$ both from the Kubo formula and from the optical Bloch equations. The pseudo-spin picture gives a nice physical description of the linear and nonlinear responses of the system as precessional modes of the pseudo-spin vector $\mathbf{S}_{\mathbf{k}}=\left(S_{x, \mathbf{k}}, S_{y, \mathbf{k}}, S_{z, \mathbf{k}}\right)$. The optical Bloch equations describe the time evolution of the pseudo-spin vector under the action of the ac electric field $\mathcal{E}_{z}$. The components of the pseudo-spin vector are the expectation values of the pseudo-spin operators

$$
\begin{aligned}
\sigma_{x, \mathbf{k}} & =d_{\mathbf{k}}^{\dagger} f_{\mathbf{k}}+f_{\mathbf{k}}^{\dagger} d_{\mathbf{k}}, \\
\sigma_{y, \mathbf{k}} & =-i\left(d_{\mathbf{k}}^{\dagger} f_{\mathbf{k}}-f_{\mathbf{k}}^{\dagger} d_{\mathbf{k}}\right), \\
\sigma_{z, \mathbf{k}} & =d_{\mathbf{k}}^{\dagger} d_{\mathbf{k}}-f_{\mathbf{k}}^{\dagger} f_{\mathbf{k}}
\end{aligned}
$$

in the ground state $|\psi\rangle$. The equations of motion for the components of $\mathbf{S}_{\mathbf{k}}$ follow from the Heisenberg equations of motion for the pseudo-spin operators $(\hbar=1, i=x, y, z)$,

$$
\dot{\sigma}_{i, \mathbf{k}}=-i\left[\sigma_{i, \mathbf{k}}, H+H_{\mathrm{int}}\right] .
$$

Working out the commutators, we find that the right-hand side contains products of pseudo-spin operators $\sigma_{i, \mathbf{k}} \sigma_{j, \mathbf{k}^{\prime}}$ $(i \neq j)$. The products occur because of the Coulomb interaction term in the Hamiltonian (11). A closed set of equations 
is obtained by replacing the average of products $\left\langle\psi\left|\sigma_{i, \mathbf{k}} \sigma_{j, \mathbf{k}^{\prime}}\right| \psi\right\rangle$ by the product of averages $\left\langle\psi\left|\sigma_{i, \mathbf{k}}\right| \psi\right\rangle\left\langle\psi\left|\sigma_{j, \mathbf{k}^{\prime}}\right| \psi\right\rangle=$ $S_{i, \mathbf{k}} S_{j, \mathbf{k}^{\prime}}$. This gives the optical Bloch equations

$$
\dot{\mathbf{S}}_{\mathbf{k}}=\left(\mathbf{H}_{\mathbf{k}}-\mathbf{M}_{\mathbf{k}}\right) \times \mathbf{S}_{\mathbf{k}},
$$

where $\mathbf{H}_{\mathbf{k}}=\left(-2 \mu_{z}\left(E_{z}+\mathcal{E}_{z}\right), 0, \varepsilon_{\mathbf{k}}-E_{f}^{\prime}\right)$ and $\mathbf{M}_{\mathbf{k}}=\frac{U}{N} \sum_{\mathbf{k}} \mathbf{S}_{\mathbf{k}}$. The symbol $\times$ represents the vector cross product. The optical Bloch equations describe the coupled motion of a collection of $N$ pseudo-spins. Each pseudo-spin precesses around a local "magnetic" field $\mathbf{H}_{\mathbf{k}}-\mathbf{M}_{\mathbf{k}}$, which is the sum of an external field $\mathbf{H}_{\mathbf{k}}$ and an average internal field $-\mathbf{M}_{\mathbf{k}}$, where $\mathbf{M}_{\mathbf{k}}$ is the pseudo-magnetization.

\section{B. Stationary solution}

In the absence of the ac electric field the optical Bloch equations have a stationary solution $\mathbf{S}_{\mathbf{k}}^{(0)}$. The stationary solution is obtained by setting $\dot{\mathbf{S}}_{\mathbf{k}}^{(0)}=0$ in Eq. (15):

$$
0=\left(\mathbf{H}_{\mathbf{k}}^{(0)}-\mathbf{M}_{\mathbf{k}}^{(0)}\right) \times \mathbf{S}_{\mathbf{k}}^{(0)} .
$$

Here $\mathbf{H}_{\mathbf{k}}^{(0)}=\left(-2 \mu_{z} E_{z}, 0, \varepsilon_{\mathbf{k}}-E_{f}^{\prime}\right)$, and $\mathbf{M}_{\mathbf{k}}^{(0)}=\frac{U}{N} \sum_{\mathbf{k}} \mathbf{S}_{\mathbf{k}}^{(0)}$. In the stationary state each pseudo-spin is lined up with the local "magnetic" field. Then there are two possibilities: $\mathbf{S}_{\mathbf{k}}^{(0)}$ is either parallel or antiparallel to $\mathbf{H}_{\mathbf{k}}^{(0)}-\mathbf{M}_{\mathbf{k}}^{(0)}$. The state with $\mathbf{S}_{\mathbf{k}}^{(0)}$ antiparallel to $\mathbf{H}_{\mathbf{k}}^{(0)}-\mathbf{M}_{\mathbf{k}}^{(0)}$ has the lower energy. Thus in the ground state all pseudo-spins point in the direction opposite the local "magnetic" field.

For zero bias field $\left(E_{z}=0\right)$, Eq. (16) is invariant under rotation about the $z$-axis. If $\mathbf{S}_{\mathbf{k}}^{(0)}$ is a solution, then so is $R_{z}(\phi) \mathbf{S}_{\mathbf{k}}^{(0)}$, where $R_{z}(\phi)$ is a rotation about the $z$-axis over an angle $\phi$. The angle $\phi$ is the phase of the gap parameter $\Delta$. For nonzero bias field, $\mathbf{S}_{\mathbf{k}}^{(0)}$ lies in the $x$ - $z$ plane and the gap parameter is real. Introducing spherical polar coordinates, $\mathbf{S}_{\mathbf{k}}^{(0)}=\left(\sin \theta_{\mathbf{k}}, 0, \cos \theta_{\mathbf{k}}\right)$ and $\mathbf{M}_{\mathbf{k}}^{(0)}=(2 \Delta, 0, U n)$. The tilting angle $\theta_{\mathbf{k}}$ is the angle between $\mathbf{S}_{\mathbf{k}}^{(0)}$ and the positive $z$-axis. The magnetization $\mathbf{M}_{\mathbf{k}}^{(0)}$ must be determined self-consistently from $\mathbf{M}_{\mathbf{k}}^{(0)}=\frac{U}{N} \sum_{\mathbf{k}} \mathbf{S}_{\mathbf{k}}^{(0)}$. The $z$-component of this equation gives Eq. (4), and the $x$-component gives Eq. (8). The stationary solution of the optical Bloch equations is the SCMF solution given in section III.

\section{Pseudo-spin precession}

To calculate the linear susceptibility from the optical Bloch equations we expand the pseudo-spin vector and pseudo-magnetization to first order in $\mathcal{E}_{z}: \mathbf{S}_{\mathbf{k}}=\mathbf{S}_{\mathbf{k}}^{(0)}+\mathbf{S}_{\mathbf{k}}^{(1)}$ and $\mathbf{M}_{\mathbf{k}}=\mathbf{M}_{\mathbf{k}}^{(0)}+\mathbf{M}_{\mathbf{k}}^{(1)}$. Also, $\mathbf{H}_{\mathbf{k}}=\mathbf{H}_{\mathbf{k}}^{(0)}+\mathbf{H}_{\mathbf{k}}^{(1)}$, with $\mathbf{H}_{\mathbf{k}}^{(1)}=\left(-2 \mu_{z} \mathcal{E}_{z}, 0,0\right)$. We substitute the expansions into the Bloch equations and collect terms of the same order in the ac electric field. To zeroth order we recover Eq. (16). To first order we get

$$
\dot{\mathbf{S}}_{\mathbf{k}}^{(1)}-\left(\mathbf{H}_{\mathbf{k}}^{(0)}-\mathbf{M}_{\mathbf{k}}^{(0)}\right) \times \mathbf{S}_{\mathbf{k}}^{(1)}-\mathbf{S}_{\mathbf{k}}^{(0)} \times \mathbf{M}_{\mathbf{k}}^{(1)}=\mathbf{H}_{\mathbf{k}}^{(1)} \times \mathbf{S}_{\mathbf{k}}^{(0)} .
$$

Taking the inner product with the stationary solution gives $\dot{\mathbf{S}}_{\mathbf{k}}^{(1)} \cdot \mathbf{S}_{\mathbf{k}}^{(0)}=0$. The ac electric field causes the pseudospin vector to precess about the stationary direction. With $\mathbf{S}_{\mathbf{k}}^{(0)}$ tilted away from the $z$-axis, the precession involves variations in all three cartesian components of $\mathbf{S}_{\mathbf{k}}$. The problem is simplified by working in the spherical polar coordinate system. In spherical polar coordinates, the stationary solution is the unit vector in the radial direction $\mathbf{e}_{r}$. The precession about $\mathbf{e}_{r}$ is decomposed into components along the polar and azimuthal units vectors $\mathbf{e}_{\theta}$ and $\mathbf{e}_{\phi}$ : $\mathbf{S}_{\mathbf{k}}^{(1)}=S_{\theta, \mathbf{k}}^{(1)} \mathbf{e}_{\theta}+S_{\phi, \mathbf{k}}^{(1)} \mathbf{e}_{\phi}$. One must remember that $\mathbf{e}_{r}$ and $\mathbf{e}_{\theta}$ vary with the tilt angle $\theta_{\mathbf{k}}$, while $\mathbf{e}_{\phi}$ is fixed since $\mathbf{S}_{\mathbf{k}}^{(0)}$ lies in the $x-z$ plane. The equations of motion for $S_{\theta, \mathbf{k}}^{(1)}$ and $S_{\phi, \mathbf{k}}^{(1)}$ are

$$
\begin{aligned}
& \dot{S}_{\theta, \mathbf{k}}^{(1)}-2 E_{\mathbf{k}} S_{\phi, \mathbf{k}}^{(1)}+M_{\phi, \mathbf{k}}^{(1)}=0 \\
& \dot{S}_{\phi, \mathbf{k}}^{(1)}+2 E_{\mathbf{k}} S_{\theta, \mathbf{k}}^{(1)}-M_{\theta, \mathbf{k}}^{(1)}=2 \mu_{z} \mathcal{E}_{z} \cos \theta_{\mathbf{k}} .
\end{aligned}
$$

Here $M_{\phi, \mathbf{k}}^{(1)}=\frac{U}{N} \sum_{\mathbf{k}^{\prime}} S_{\phi, \mathbf{k}^{\prime}}^{(1)}$ and $M_{\theta, \mathbf{k}}^{(1)}=\frac{U}{N} \sum_{\mathbf{k}^{\prime}} \cos \left(\theta_{\mathbf{k}}-\theta_{\mathbf{k}^{\prime}}\right) S_{\theta, \mathbf{k}^{\prime}}^{(1)}$. The appearance of the cosine factor in $M_{\theta, \mathbf{k}}^{(1)}$ is due to the variation of $\mathbf{e}_{\theta}$ with $\theta_{\mathbf{k}}$. In deriving Eqs. (18) and (19) from Eq. (17) we have used the fact that $S_{r, \mathbf{k}}^{(1)}=0$, which follows from $\dot{S}_{r, \mathbf{k}}^{(1)}=0$ and the initial condition $S_{r, \mathbf{k}}^{(1)}(t=0)=0$. 


\section{Noninteracting quasiparticles}

It is instructive to first calculate the absorption spectrum neglecting the Coulomb interaction between the optically excited quasiparticles. In the pseudo-spin picture, this corresponds to setting $M_{\phi, \mathbf{k}}^{(1)}=M_{\theta, \mathbf{k}}^{(1)}=0$ in Eqs. (18) and (19). The linear susceptibility is $\chi_{z z}^{(1)}=P_{z}^{(1)} / \mathcal{E}_{z}$, where $P_{z}^{(1)}=\left(N \mu_{z} / \Omega\right) \sum_{\mathbf{k}} S_{\theta, \mathbf{k}}^{(1)} \cos \theta_{\mathbf{k}}$ is the first-order polarization. Solving Eqs. (18) and (19) for $S_{\theta, \mathbf{k}}^{(1)}$, we obtain

$$
\chi_{z z}^{(1)}=-\frac{N \mu_{z}^{2}}{\Omega} \sum_{\mathbf{k}}\left(\frac{n_{\mathbf{k}, \mathbf{k}}^{2}}{\omega-2 E_{\mathbf{k}}}-\frac{n_{\mathbf{k}, \mathbf{k}}^{2}}{\omega+2 E_{\mathbf{k}}}\right),
$$

where $n_{\mathbf{k}, \mathbf{k}^{\prime}}=u_{\mathbf{k}} u_{\mathbf{k}^{\prime}}-v_{\mathbf{k}} v_{\mathbf{k}^{\prime}}$ is a coherence factor. The frequency $\omega$ is understood to have a small positive imaginary part $\delta$. The physical origin of the coherence factor $n_{\mathbf{k}, \mathbf{k}^{\prime}}$ can be understood as follows 20]: In the absorption process, an incoming photon of momentum $\mathbf{k}-\mathbf{k}^{\prime}$ creates a quasi-electron of momentum $\mathbf{k}$ and a quasi-hole of momentum $-\mathbf{k}^{\prime}$. This can be done in two different ways: (1) If the pair states $(\mathbf{k},-\mathbf{k})$ and $\left(\mathbf{k}^{\prime},-\mathbf{k}^{\prime}\right)$ are initially empty, by adding a $d$-electron in $\mathbf{k}$ and adding an $f$-hole in $-\mathbf{k}^{\prime}$. This process has amplitude $u_{\mathbf{k}} u_{\mathbf{k}^{\prime}}$. (2) If the pair states (k, $\left.-\mathbf{k}\right)$ and $\left(\mathbf{k}^{\prime},-\mathbf{k}^{\prime}\right)$ are initially occupied, by removing an $f$-hole from $-\mathbf{k}$ and removing a $d$-electron from $\mathbf{k}^{\prime}$. This process has amplitude $-v_{\mathbf{k}} v_{\mathbf{k}^{\prime}}$. The overall amplitude for the creation process is $u_{\mathbf{k}} u_{\mathbf{k}^{\prime}}-v_{\mathbf{k}} v_{\mathbf{k}^{\prime}}=n_{\mathbf{k}, \mathbf{k}^{\prime}}$.

For the model system the absorption spectrum can be found analytically. When the $f$-level lies inside the $d$-band $\left(\left|E_{f}\right| \leq W\right)$, the energy gap is $2 \Delta$, and the absorption rises as $\sqrt{\omega-2 \Delta}$ above threshold. When the $f$-level lies outside the $d$-band $\left(\left|E_{f}\right|>W\right)$, the gap is $\sqrt{\left(\left|E_{f}\right|-W\right)^{2}+4 \Delta^{2}}$, and there is a discontinuous jump in the absorption at threshold. The single-quasiparticle result for the absorption spectrum at half-filling $\left(E_{f}=0\right)$ is shown by the dash-dotted line in Fig. 5 .

\section{E. Final-state interaction}

The quasiparticles created in the optical transition interact via the Coulomb potential $U$. In the normal state $(\Delta=0)$ the final-state interaction leads to the Wannier exciton. In the mixed-valent state $(\Delta>0)$ the final-state interaction leads to ferroelectric resonance and a threshold singularity in the infrared absorption spectrum. In the pseudo-spin picture, the final-state interaction corresponds to $M_{\phi, \mathbf{k}}^{(1)}$ and $M_{\theta, \mathbf{k}}^{(1)}$. For a separable Coulomb potential, Eqs. (18) and (19) can be solved analytically. The pseudo-spin components are given by

$$
\begin{aligned}
S_{\theta, \mathbf{k}}^{(1)} & =\mu_{z} \mathcal{E}_{z}\left(\frac{\Gamma(\mathbf{k}, \omega)}{\omega-2 E_{\mathbf{k}}}-\frac{\Gamma(\mathbf{k},-\omega)}{\omega+2 E_{\mathbf{k}}}\right), \\
S_{\phi, \mathbf{k}}^{(1)} & =-i \mu_{z} \mathcal{E}_{z}\left(\frac{\Gamma(\mathbf{k}, \omega)}{\omega-2 E_{\mathbf{k}}}+\frac{\Gamma(\mathbf{k},-\omega)}{\omega+2 E_{\mathbf{k}}}\right),
\end{aligned}
$$

where

$$
\begin{aligned}
\Gamma(\mathbf{k}, \omega)= & -\cos \theta_{\mathbf{k}}-\frac{U}{N} \sum_{\mathbf{k}^{\prime}} \frac{\cos ^{2}\left(\frac{\theta_{k}-\theta_{k^{\prime}}}{2}\right) \Gamma\left(\mathbf{k}^{\prime}, \omega\right)}{\omega-2 E_{\mathbf{k}^{\prime}}} \\
& -\frac{U}{N} \sum_{\mathbf{k}^{\prime}} \frac{\sin ^{2}\left(\frac{\theta_{k}-\theta_{k^{\prime}}}{2}\right) \Gamma\left(\mathbf{k}^{\prime},-\omega\right)}{\omega+2 E_{\mathbf{k}^{\prime}}}
\end{aligned}
$$

is a vertex function. In diagrammatic terms, the vertex function is the sum of all ladder diagrams contributing to the propagation of the quasiparticle pair.

In the normal state $\left(\theta_{\mathbf{k}}=\pi\right)$ Eq. (23) yields the Wannier exciton. The pole of the vertex function gives the exciton binding energy $E_{\mathrm{b}}=W \operatorname{coth}(W / U)-W$. The absorption spectrum of the normal state consists of an exciton line at $\omega=E_{g}-E_{b}$, and a continuum between $\omega=E_{g}$ and $\omega=E_{g}+2 W$, where $E_{g}=-W-E_{f}$ is the $d-f$ band gap. The final-state interaction enhances the absorption at $\omega=E_{g}$.

In the mixed-valent state the vertex function has three components:

$$
\Gamma(\mathbf{k}, \omega)=-\cos \theta_{\mathbf{k}} \Gamma_{1}(\omega)+\sin \theta_{\mathbf{k}} \Gamma_{2}(\omega)+\Gamma_{3}(\omega) .
$$

The components $\Gamma_{1}(\omega)$ and $\Gamma_{2}(\omega)$ are even in $\omega$, while $\Gamma_{3}(\omega)$ is odd. Substitution of Eq. 24) into Eq. 23. yields a set of of three algebraic equations for the vertex components: 


$$
\left(\begin{array}{ccc}
\left(\omega^{2}-M^{2}\right) A(\omega)+R & M B(\omega) & \omega B(\omega) \\
M B(\omega) & 1+M^{2} A(\omega) & \omega M A(\omega) \\
\omega B(\omega) & \omega M A(\omega) & \omega^{2} A(\omega)+R
\end{array}\right)\left(\begin{array}{l}
\Gamma_{1}(\omega) \\
\Gamma_{2}(\omega) \\
\Gamma_{3}(\omega)
\end{array}\right)=\left(\begin{array}{l}
1 \\
0 \\
0
\end{array}\right)
$$

Here $M=2 \Delta+\mu_{z} E_{z}$ and $R=\mu_{z} E_{z} /\left(\Delta+\mu_{z} E_{z}\right)$. The functions $A(\omega)$ and $B(\omega)$ are given by

$$
\begin{aligned}
& A(\omega)=\frac{U}{N} \sum_{\mathbf{k}} \frac{1}{2 E_{\mathbf{k}}\left(\omega-2 E_{\mathbf{k}}\right)\left(\omega+2 E_{\mathbf{k}}\right)}, \\
& B(\omega)=\frac{U}{N} \sum_{\mathbf{k}} \frac{\varepsilon_{\mathbf{k}}-E_{f}}{2 E_{\mathbf{k}}\left(\omega-2 E_{\mathbf{k}}\right)\left(\omega+2 E_{\mathbf{k}}\right)} .
\end{aligned}
$$

For the model system $A(\omega)$ and $B(\omega)$ can found analytically. The explicit expressions are given in the Appendix. Solving Eq. (25) for $\Gamma_{1}(\omega)$ gives

$$
\Gamma_{1}(\omega)=\frac{\left(\omega^{2}+R M^{2}\right) A(\omega)+R}{\left[\left(\omega^{2}-M^{2}\right) A(\omega)+R\right]\left[\left(\omega^{2}+R M^{2}\right) A(\omega)+R\right]-\left(\omega^{2}+R M^{2}\right) B^{2}(\omega)} .
$$

The linear susceptibility is

$$
\chi_{z z}^{(1)}(\omega)=\frac{2 N \mu_{z}^{2}}{\Omega U}\left(\Gamma_{1}(\omega)-1\right)
$$

\section{F. Infrared absorption spectrum}

We first consider the absorption spectrum in the absence of a static electric field. We calculate the continuum absorption in zero bias field, setting $M=2 \Delta$ and $R=0$ in Eq. (28). For an energy gap of several meV, the continuum lies in the far infrared. The linear susceptibility in zero bias field is

$$
\chi_{z z}^{(1)}(\omega)=\frac{2 N \mu_{z}^{2}}{\Omega U}\left(\frac{A(\omega)}{\left(\omega^{2}-4 \Delta^{2}\right) A^{2}(\omega)-B^{2}(\omega)}-1\right) .
$$

The solid lines in Fig. 3 show the imaginary part of Eq. (30) for several values of $E_{f}$. When the $f$-level lies far below the bottom of the $d$-band (top curve), the continuum absorption of the mixed-valent state is very similar to the continuum absorption of the normal state. There is a slight enhancement near the energy gap. As the $f$-level approaches the bottom of the $d$-band, the enhancement becomes more and more pronounced. When the $f$-level lies within the $d$-band, the spectrum has a threshold singularity at $\omega=2 \Delta$. The spectrum for $E_{f}$ above the center of the $d$-band is the same as the spectrum for $-E_{f}$ below it. From Eq. (30) we find that when $\left|E_{f}\right|<W$, the threshold singularity is $\epsilon^{-1 / 2} \theta(\epsilon)$, and when $\left|E_{f}\right|=W$ the singularity is $\epsilon^{-1 / 2} \ln ^{-2}(\epsilon) \theta(\epsilon)$, where $\epsilon=\omega-2 \Delta$. When the $f$-level lies outside the $d$-band the singularity is cut off because the energy gap is larger than $2 \Delta$. The singularity is due to the final-state interaction between the optically excited quasiparticles. In the single-quasiparticle treatment, the absorption rises continuously from zero according to $\epsilon^{1 / 2} \theta(\epsilon)$. The singularity is not an artifact of the simple model, and should be observable in real materials. Roundoff may occur due to lifetime effects and sample inhomogeneities.

\section{G. Ferroelectric resonance}

The dash-dotted line in Fig. 3 represents the absorption spectrum of the mixed-valent system in a bias field. The spectrum consists of two parts: a peak at $\omega=\omega_{0}$, and a continuum above $\omega=2 \Delta$. We shall show that $\omega_{0}$ is the ferroelectric resonance frequency. The continuum part of the absorption spectrum is blue-shifted only slightly by the bias field.

Ferroelectric resonance occurs when an electronic ferroelectric, placed in a bias field $E_{z}$, is acted upon by an alternating field $\mathcal{E}_{z}$ of frequency $\omega_{0}$. The ferroelectric resonance frequency $\omega_{0}$ is the frequency of the Goldstone mode in the bias field $E_{z}$. The Goldstone mode corresponds to a uniform precession of the pseudo-spins around the $z$-axis. For an ideal system $\omega_{0}$ vanishes at zero bias field. For a real system the external bias field $E_{z}$ must be replaced by an effective internal field $E_{\text {eff }}$, which may depend on the sample shape, the domain structure, and the crystal anisotropy. An example of a contribution to $E_{\text {eff }}$ is the depolarization field $-N_{z} P_{z}$ of the sample boundary, where $N_{z}$ is the 
depolarization factor. The effective field remains nonzero in the absence of the external bias field, yielding a finite resonance frequency for an unbiased sample.

Ferroelectric resonance is the electric analogue of ferromagnetic resonance in a ferromagnetic insulator. In the magnetic case, the alternating field causes a uniform precession of real spins around the $z$-direction. The ferromagnetic resonance frequency is $\omega_{0}=\gamma H_{z}$, where $\gamma$ is the gyromagnetic ratio, and $H_{z}$ is the bias field. In real samples $H_{z}$ must be replaced by an effective field $H_{\text {eff }}$ depending on the sample geometry, magnetic domain structure, and crystal magnetic anisotropy [21].

Ferroelectric resonance does not occur in displacive ferroelectrics because the order parameter only has a discrete symmetry. This means there is no Goldstone mode in the excitation spectrum of a displacive ferroelectric. Ferroelectric liquid crystals $d o$ have an order parameter with a continuous symmetry. The dielectric response of the Goldstone mode has been observed [22] in ferroelectric liquid crystals by means of broadband dielectric spectroscopy.

We now calculate the dependence of the ferroelectric resonance frequency of an ideal system on the external bias field $E_{z}$. For a real system, $E_{z}$ must be replaced by $E_{\text {eff }}$. The ferroelectric resonance frequency is given by the equation $D\left(\omega_{0}\right)=0$, where $D(\omega)$ is the denominator of Eq. (28). For $\mu_{z} E_{z}$ small compared to $\Delta$, an approximate solution may be obtained by expanding $D\left(\omega_{0}\right)$ in a Taylor series around $\omega_{0}=0: D\left(\omega_{0}\right)=D(0)+\frac{1}{2} \omega_{0}^{2} D^{\prime \prime}(0)$. The linear term vanishes because $D(\omega)$ is even in $\omega$. Neglecting terms of order $R^{2}$ in $D(0)$ and of order $R$ in $D^{\prime \prime}(0)$, we find

$$
\omega_{0}=\left[-4 \mu_{z} \Delta\left(1+\frac{A(0)}{4 \Delta^{2} A^{2}(0)+B^{2}(0)}\right)\right]^{1 / 2} \sqrt{E_{z}}
$$

Substituting the explicit expressions for $A(0)$ and $B(0)$ given in the Appendix yields

$$
\omega_{0}=\left(\frac{4 \mu_{z} \Delta E_{c}^{\prime}}{U}\right)^{1 / 2} \sqrt{E_{z}}
$$

where $E_{c}^{\prime}=E_{c}-U$ is the critical value of $E_{f}^{\prime}$. A useful estimate of $\omega_{0}$ is the arithmetic mean of the gap $2 \Delta$ and the field energy $\mu_{z} E_{z}$. For $\mu_{z}=10^{-29} \mathrm{Cm}, 2 \Delta=1 \mathrm{meV}$, and $E_{z}$ between $10 \mathrm{~V} / \mathrm{cm}$ and $10^{4} \mathrm{~V} / \mathrm{cm}, \omega_{0}$ is between 0.01 $\mathrm{meV}$ and $1 \mathrm{meV}$, i.e. in the microwave regime.

The condition $D\left(\omega_{0}\right)=0$ is not sufficient for a peak in the absorption spectrum at $\omega=\omega_{0}$. One must also have $N\left(\omega_{0}\right) \neq 0$, where $N(\omega)$ is the numerator of Eq. (28). The strength of the pole at $\omega_{0}$ is $Z_{0}=-\pi N\left(\omega_{0}\right) / D^{\prime}\left(\omega_{0}\right)$. Away from half-filling, one finds $Z_{0}>0$. Exactly at half-filling, $B(\omega)=0$. As can be seen at once from Eq. (28), we then have $Z_{0}=0$. The zero strength at half-filling is an artifact of our simple model, which has $\rho(\epsilon)=\rho(-\epsilon)$. For a realistic $d$-band there will be a resonance peak at half-filling.

An important problem in ferromagnetic resonance is to account for the width of the ferromagnetic resonance line [23]. The line width is due to the relaxation of the uniform precession by spin-spin and spin-lattice interactions. Various damping terms may be added to the Bloch equations to describe the relaxation. A possible damping term is $-\left(\mathbf{S}_{\mathbf{k}}-\mathbf{S}_{\mathbf{k}}^{(0)}\right) / \tau$, where $\tau$ is a phenomenological relaxation time. In the equation of motion for $S_{\mathbf{k}}^{(1)}$, this leads to the replacement of $d / d t$ by $d / d t+1 / \tau$. Thus, the simplest approach to damping (the one we have adopted here) is to add an imaginary part $\delta=1 / \tau$ to $\omega$. A detailed study of the width of the ferroelectric resonance line is left for future research.

\section{SECOND-HARMONIC GENERATION}

Second-harmonic generation is the generation of an outgoing electromagnetic wave of frequency $2 \omega$ from two incoming waves of frequency $\omega$. For incoming waves propagating along $\mathbf{k}_{1}$ and $\mathbf{k}_{2}$, the second-harmonic radiation is most effectively generated in the phase-matching direction $\mathbf{k}_{1}+\mathbf{k}_{2}$. In this direction both energy and momentum are conserved.

The ability of a medium to sustain second-harmonic generation is characterized by the second-harmonic susceptibility tensor $\chi_{i j k}^{(2)}(2 \omega, \omega, \omega)$. Here $i, j, k=x, y, z$ are the Cartesian indices. The polarization $P_{i}(2 \omega)$ induced in the medium by incoming fields $\mathcal{E}_{j}(\omega)$ and $\mathcal{E}_{k}(\omega)$ is given by $P_{i}(2 \omega)=\chi_{i j k}^{(2)}(2 \omega, \omega, \omega) \mathcal{E}_{j}(\omega) \mathcal{E}_{k}(\omega)$. For a medium with inversion symmetry, the second-harmonic susceptibility must satisfy $\chi_{i j k}^{(2)}=-\chi_{i j k}^{(2)}=0$. Therefore, second-harmonic generation cannot occur in media with inversion symmetry.

In the mixed-valent system, the inversion symmetry is spontaneously broken by the pairing of electronic states of opposite parity. This leads to the appearance of a built-in polarization in the $z$-direction. If the incoming fields have a 
component along the $z$-axis, second-harmonic generation can occur. We calculate the second-harmonic susceptibility $\chi_{z z z}^{(2)}$ for incoming fields polarized along the $z$-axis. For incoming fields polarized at an angle $\phi$ relative to the $z$-axis, the second-harmonic susceptibility is reduced by $\cos ^{2} \phi$.

\section{A. Pseudo-spin nutation}

We calculate the second-harmonic susceptibility $\chi_{z z z}^{(2)}$ from the optical Bloch equations by expanding $\mathbf{S}_{\mathbf{k}}$ and $\mathbf{M}_{\mathbf{k}}$ to second order in $\mathcal{E}_{z}$. The external "magnetic" field $\mathbf{H}_{\mathbf{k}}$ has no components of second or higher order. The equation of motion for $\mathbf{S}_{\mathbf{k}}^{(2)}$ is

$$
\dot{\mathbf{S}}_{\mathbf{k}}^{(2)}-\left(\mathbf{H}_{\mathbf{k}}^{(0)}-\mathbf{M}_{\mathbf{k}}^{(0)}\right) \times \mathbf{S}_{\mathbf{k}}^{(2)}+\mathbf{M}_{\mathbf{k}}^{(2)} \times \mathbf{S}_{\mathbf{k}}^{(0)}=\left(\mathbf{H}_{\mathbf{k}}^{(1)}-\mathbf{M}_{\mathbf{k}}^{(1)}\right) \times \mathbf{S}_{\mathbf{k}}^{(1)} .
$$

Eq. (33) has the same form as Eq. (17), except with a more complicated right hand side. As before, we decompose $\mathbf{S}_{\mathbf{k}}^{(2)}$ into its radial, polar, and azimuthal components: $\mathbf{S}_{\mathbf{k}}^{(2)}=S_{r, \mathbf{k}}^{(2)} \mathbf{e}_{r}+S_{\theta, \mathbf{k}}^{(2)} \mathbf{e}_{\theta}+S_{\phi, \mathbf{k}}^{(2)} \mathbf{e}_{\phi}$. To second order, the pseudo-spin has a nonzero radial component $S_{r, \mathbf{k}}^{(2)}$. This means the motion is no longer a regular precession: the pseudo-spin nutates during the precession. (Nutation is the up-and-down motion of the precession axis.) The nutation frequency is twice the precession frequency. This can be shown as follows: the equation of motion for $S_{r, \mathbf{k}}^{(2)}$ is obtained by taking the inner product of Eq. (33) with $\mathbf{e}_{r}$. Using the vector identity $(\mathbf{A} \times \mathbf{B}) \cdot \mathbf{C}=(\mathbf{C} \times \mathbf{A}) \cdot \mathbf{B}$ and taking the inner product of Eq. (17) with $\mathbf{e}_{r}$ shows that the equation for $S_{r, \mathbf{k}}^{(2)}$ can be written as $\dot{S}_{r, \mathbf{k}}^{(2)}=-\dot{\mathbf{S}}_{\mathbf{k}}^{(1)} \cdot \mathbf{S}_{\mathbf{k}}^{(1)}$. Upon integration, we find

$$
S_{r, \mathbf{k}}^{(2)}=-\frac{1}{2}\left(S_{\theta, \mathbf{k}}^{(1)} S_{\theta, \mathbf{k}}^{(1)}+S_{\phi, \mathbf{k}}^{(1)} S_{\phi, \mathbf{k}}^{(1)}\right) .
$$

The equations of motion for $S_{\theta, \mathbf{k}}^{(2)}$ and $S_{\phi, \mathbf{k}}^{(2)}$ are obtained by taking the inner product of Eq. (33) with $\mathbf{e}_{\theta}$ and $\mathbf{e}_{\phi}$ respectively. Apart from the more complicated source terms, a new feature occuring in second order is that $S_{r, \mathbf{k}}^{(2)}$ is now nonzero. This leads to an additional term $\frac{U}{N} \sum_{\mathbf{k}^{\prime}} \sin \left(\theta_{\mathbf{k}}-\theta_{\mathbf{k}^{\prime}}\right) S_{r, \mathbf{k}^{\prime}}^{(2)}$ on the left hand side of the equation for $S_{\phi, \mathbf{k}}^{(2)}$. However, since we have already solved for $S_{r, \mathbf{k}}^{(2)}$ in Eq. (34), the additional term can be taken over to the right hand side and treated as an extra source term. Combining all source terms into driving forces $F_{\theta, \mathbf{k}}^{(2)}$ and $F_{\phi, \mathbf{k}}^{(2)}$, the equations of motion for $S_{\theta, \mathbf{k}}^{(2)}$ and $S_{\phi, \mathbf{k}}^{(2)}$ are

$$
\begin{array}{r}
\dot{S}_{\theta, \mathbf{k}}^{(2)}-2 E_{\mathbf{k}} S_{\phi, \mathbf{k}}^{(2)}+M_{\phi, \mathbf{k}}^{(2)}=F_{\theta, \mathbf{k}}^{(2)} \\
\dot{S}_{\phi, \mathbf{k}}^{(2)}+2 E_{\mathbf{k}} S_{\theta, \mathbf{k}}^{(2)}-M_{\theta, \mathbf{k}}^{(2)}=F_{\phi, \mathbf{k}}^{(2)}
\end{array}
$$

with

$$
\begin{aligned}
& F_{\theta, \mathbf{k}}^{(2)}=\left(2 \mu_{z} \mathcal{E}_{z} \sin \theta_{\mathbf{k}}+M_{r, \mathbf{k}}^{(1)}\right) S_{\phi, \mathbf{k}}^{(1)} \\
& F_{\phi, \mathbf{k}}^{(2)}=-\left(2 \mu_{z} \mathcal{E}_{z} \sin \theta_{\mathbf{k}}+M_{r, \mathbf{k}}^{(1)}\right) S_{\theta, \mathbf{k}}^{(1)}-\frac{U}{N} \sum_{\mathbf{k}^{\prime}} \sin \left(\theta_{\mathbf{k}}-\theta_{\mathbf{k}^{\prime}}\right) S_{r, \mathbf{k}^{\prime}}^{(2)}
\end{aligned}
$$

Here $M_{r, \mathbf{k}}^{(1)}=\frac{U}{N} \sum_{\mathbf{k}^{\prime}} \sin \left(\theta_{\mathbf{k}}-\theta_{\mathbf{k}^{\prime}}\right) S_{\theta, \mathbf{k}^{\prime}}^{(1)}$. A very important observation is that $\operatorname{since} \sin \theta_{\mathbf{k}}=\Delta / E_{\mathbf{k}}$, all source terms are proportional to $\Delta$. The second-harmonic susceptibility vanishes identically when $\Delta=0$.

\section{B. Independent quasiparticles}

We first calculate the second-harmonic susceptibility neglecting the Coulomb interaction between the optically excited quasiparticles. This corresponds to setting $M_{\theta, \mathbf{k}}^{(2)}=M_{\phi, \mathbf{k}}^{(2)}=0$ on the left hand side of Eqs. (35) and (36), and $F_{\theta, \mathbf{k}}^{(2)}=2 \mu_{z} \mathcal{E}_{z} S_{\phi, \mathbf{k}}^{(1)} \sin \theta_{\mathbf{k}}$ and $F_{\phi, \mathbf{k}}^{(2)}=-2 \mu_{z} \mathcal{E}_{z} S_{\theta, \mathbf{k}}^{(1)} \sin \theta_{\mathbf{k}}$ on the right hand side. The second-harmonic susceptibility is $\chi_{z z z}^{(2)}=P_{z}^{(2)} / \mathcal{E}_{z}^{2}$, where $P_{z}^{(2)}=\left(N \mu_{z} / \Omega\right) \sum_{\mathbf{k}}\left(S_{\theta, \mathbf{k}}^{(2)} \cos \theta_{\mathbf{k}}+S_{r, \mathbf{k}}^{(2)} \sin \theta_{\mathbf{k}}\right)$ is the second-order polarization. The secondorder polarization has a contribution from the radial component $S_{r, \mathbf{k}}^{(2)}$. Solving Eqs. (35) and (36) for $S_{\theta, \mathbf{k}}^{(2)}$, and using Eq. (34) for $S_{r, \mathbf{k}}^{(2)}$, we obtain 


$$
\begin{aligned}
\chi_{z z z}^{(2)}= & -\frac{N \mu_{z}^{3}}{\Omega} \sum_{\mathbf{k}}\left(\frac{2 m_{\mathbf{k}, \mathbf{k}} n_{\mathbf{k}, \mathbf{k}}^{2}}{\left(\omega-2 E_{\mathbf{k}}\right)\left(2 \omega-2 E_{\mathbf{k}}\right)}\right. \\
& \left.+\frac{2 m_{\mathbf{k}, \mathbf{k}} n_{\mathbf{k}, \mathbf{k}}^{2}}{\left(\omega+2 E_{\mathbf{k}}\right)\left(2 \omega+2 E_{\mathbf{k}}\right)}-\frac{2 m_{\mathbf{k}, \mathbf{k}} n_{\mathbf{k}, \mathbf{k}}^{2}}{\left(\omega-2 E_{\mathbf{k}}\right)\left(\omega+2 E_{\mathbf{k}}\right)}\right) .
\end{aligned}
$$

The coherence factor $m_{\mathbf{k}, \mathbf{k}^{\prime}}$ is given by $m_{\mathbf{k}, \mathbf{k}^{\prime}}=u_{\mathbf{k}} v_{\mathbf{k}^{\prime}}+v_{\mathbf{k}} u_{\mathbf{k}^{\prime}}$. The physical origin of the coherence factor $m_{\mathbf{k}, \mathbf{k}^{\prime}}$ is the scattering of a quasiparticle by the second incoming photon. The second photon can either scatter the quasi-electron from $\mathbf{k}$ to $\mathbf{k}^{\prime}$, or the quasi-hole from $-\mathbf{k}^{\prime}$ to $-\mathbf{k}$. The overall amplitude for the scattering process is $2 m_{\mathbf{k}, \mathbf{k}^{\prime}}$. Since $m_{\mathbf{k}, \mathbf{k}}=\Delta / E_{\mathbf{k}}$, the second-harmonic susceptibility is directly proportional to $\Delta$.

\section{Final-state interaction}

For a separable Coulomb potential, an analytic solution for the second-harmonic susceptibility including the finalstate interaction is possible in principle. However, the large number of driving terms in Eqs. (37) and (38) presents a considerable challenge. We have instead approached the problem numerically. This is done in analogy with the classical mechanics treatment of forced oscillations. The azimuthal component $S_{\phi, \mathbf{k}}$ is the generalized coordinate $q_{\mathbf{k}}$, and the polar component $S_{\theta, \mathbf{k}}$ is the negative of the conjugate momentum $p_{\mathbf{k}}$. Eqs. (35) and (36) are the Hamilton equations of motion for $q_{\mathbf{k}}$ and $p_{\mathbf{k}}$. The matrices $T$ and $V$ are

$$
\begin{aligned}
T_{\mathbf{k}, \mathbf{k}^{\prime}}^{-1} & =2 E_{\mathbf{k}} \delta_{\mathbf{k}, \mathbf{k}^{\prime}}-\frac{U}{N} \cos \left(\theta_{\mathbf{k}}-\theta_{\mathbf{k}^{\prime}}\right), \\
V_{\mathbf{k}, \mathbf{k}^{\prime}} & =2 E_{\mathbf{k}} \delta_{\mathbf{k}, \mathbf{k}^{\prime}}-\frac{U}{N} .
\end{aligned}
$$

The first step is to find the normal modes of oscillation of the system of pseudo-spins. The normal-mode equations are

$$
\sum_{\mathbf{k}^{\prime}} V_{\mathbf{k}, \mathbf{k}^{\prime}} A_{\mathbf{k}^{\prime}, n}=\left(2 E_{n}\right)^{2} \sum_{\mathbf{k}^{\prime}} T_{\mathbf{k}, \mathbf{k}^{\prime}} A_{\mathbf{k}^{\prime}, n}
$$

where $2 E_{n}$ is the frequency of normal mode $n$, and $A_{\mathbf{k}, n}$ is the amplitude of $S_{\phi, \mathbf{k}}$ in the normal mode $n$. Since $T$ and $V$ are both real and symmetric, the frequencies are all real and positive. There is one Goldstone mode, whose frequency is the ferroelectric resonance frequency $\omega_{0}$. The remaining $N-1$ frequencies form a continuum above the energy gap.

The second step is to obtain the forced oscillation of $S_{\theta, \mathbf{k}}^{(2)}$ and $S_{\phi, \mathbf{k}}^{(2)}$ when driven by $F_{\theta, \mathbf{k}}^{(2)}$ and $F_{\phi, \mathbf{k}}^{(2)}$. This is done by solving for the motion in normal coordinates, and then taking linear combinations to obtain the motion in the original coordinates. The force driving the normal coordinate $\zeta_{n}$ has frequency $2 \omega$ and amplitude $Q_{n}=-\sum_{\mathbf{k}}\left(A_{\mathbf{k}, n} F_{\theta, \mathbf{k}}^{(2)}+\right.$ $\left.2 i \omega A_{n, \mathbf{k}}^{-1} F_{\phi, \mathbf{k}}^{(2)}\right)$. This causes the normal coordinate to oscillate with frequency $2 \omega$ and amplitude $\zeta_{n}=Q_{n} /\left(4 E_{n}^{2}-4 \omega^{2}\right)$. The original coordinates oscillate with frequency $2 \omega$ and amplitudes $S_{\phi, \mathbf{k}}^{(2)}=\sum_{n} A_{\mathbf{k}, n} \zeta_{n}$ and $S_{\theta, \mathbf{k}}^{(2)}=2 i \omega \sum_{n} A_{\mathbf{k}, n}^{-1} \zeta_{n}+$ $\sum_{\mathbf{k}^{\prime}} T_{\mathbf{k}, \mathbf{k}^{\prime}} F_{\phi, \mathbf{k}^{\prime}}^{(2)}$. The complete motion in the original coordinates is $(m=2)$ :

$$
\begin{aligned}
S_{\phi, \mathbf{k}}^{(m)} & =\sum_{n, \mathbf{k}^{\prime}} \frac{A_{\mathbf{k}, n}\left(A_{\mathbf{k}^{\prime}, n} F_{\theta, \mathbf{k}^{\prime}}^{(m)}+m i \omega A_{n, \mathbf{k}^{\prime}}^{-1} F_{\phi, \mathbf{k}^{\prime}}^{(m)}\right)}{m^{2} \omega^{2}-4 E_{n}^{2}}, \\
S_{\theta, \mathbf{k}}^{(m)} & =\sum_{n, \mathbf{k}^{\prime}} \frac{A_{n, \mathbf{k}}^{-1}\left(4 E_{n}^{2} A_{n, \mathbf{k}^{\prime}}^{-1} F_{\phi, \mathbf{k}^{\prime}}^{(m)}-m i \omega A_{\mathbf{k}^{\prime}, n} F_{\theta, \mathbf{k}^{\prime}}^{(m)}\right)}{4 E_{n}^{2}-m^{2} \omega^{2}} .
\end{aligned}
$$

Here we have used the orthonormality condition $\delta_{n, n^{\prime}}=\sum_{\mathbf{k}, \mathbf{k}^{\prime}} A_{\mathbf{k}, n} T_{\mathbf{k}, \mathbf{k}^{\prime}} A_{\mathbf{k}^{\prime}, n^{\prime}}$ for the matrix of eigenvectors $A_{\mathbf{k}, n}$. Eqs. (43) and (44) apply to $m$-th harmonic generation in general.

The computational task is summarized as follows: First compute $S_{\phi, \mathbf{k}}^{(1)}$ and $S_{\theta, \mathbf{k}}^{(1)}$ from Eqs. (43) and (44) with $m=1$ and $F_{\theta, \mathbf{k}}^{(1)}=0, F_{\phi, \mathbf{k}}^{(1)}=2 \mu_{z} \mathcal{E}_{z} \cos \theta_{\mathbf{k}}$. To avoid a singular denominator, $\omega$ is given a small positive imaginary part $\delta$. Then compute $S_{r, \mathbf{k}}^{(2)}$ from Eq. (34), and $F_{\theta, \mathbf{k}}^{(2)}$ and $F_{\phi, \mathbf{k}}^{(2)}$ from Eqs. (37) and (38). Finally, compute $S_{\theta, \mathbf{k}}^{(2)}$ from Eq. (44) with $m=2$. 
The results of the calculation are shown in Fig. 4 . The figure shows the amplitude $\left|\chi_{z z z}^{(2)}(2 \omega, \omega, \omega)\right|$ of the secondharmonic susceptibility as a function of the photon energy $\omega$, for several values of $E_{f}$, in zero bias field. The important features are: (1) The second-harmonic susceptibility is directly proportional to $\Delta$. (2) When the $f$-level lies inside the $d$-band, the second-harmonic conversion efficiency is strongly enhanced at $\omega=\Delta$, and less strongly at $\omega=2 \Delta$. The first feature shows that second-harmonic generation can be used to test the validity of the SCMF solution in real mixed-valent compounds. The second feature distinguishes the single-quasiparticle treatment of the second-harmonic response from the SCMF treatment. The enhancement of the conversion efficiency is due to the final-state interaction between the optically excited quasiparticles.

\section{EXISTING EXPERIMENTAL EVIDENCE AND PROPOSED TESTS}

As experimental tests of electronic ferroelectricity in a mixed-valent compound we propose measurements of the static dielectric constant, the microwave absorption spectrum, and the second-harmonic susceptibility. As an example, consider $\mathrm{SmB}_{6}$. The crystal structure of $\mathrm{SmB}_{6}$ has cubic symmetry, with $\mathrm{B}_{6}$ octahedra at the body center, and $\mathrm{Sm}$ ions at the corners of a conventional bcc unit cell with lattice constant $a=4.13 \AA$. The crystal has inversion symmetry at the bcc lattice points. Through measurements of the ionic radius, the valence of the $\mathrm{Sm}$ ion is found to be 2.53 , almost halfway between 2 and 3 , so that the $f$-level lies near the center of the conduction band.

The measured far-infrared absorption spectrum [14 16] of $\mathrm{SmB}_{6}$ can be interpreted in accordance with the SCMF solution. In Fig. 5 we compare the mean-field and single-quasiparticle results for the linear susceptibility to experimental data on $\mathrm{SmB}_{6}$ taken from Ref. [14]. The data show an energy gap around $2 \Delta=4 \mathrm{meV}$, and a sharp peak at threshold. The mean-field theory fits the data very well in the threshold region, whereas the single-quasiparticle theory gives a qualitatively wrong behaviour. Away from threshold, discrepancies between mean-field theory and experiment occur because of our simple model. Further experimental indication of the validity of the SCMF solution in $\mathrm{SmB}_{6}$ is provided by the electron tunneling spectrum [17], which can be interpreted by analogy with Giaever tunneling in a superconductor.

Ref. 114 also reports a measurement of the static dielectric constant of $\mathrm{SmB}_{6}$ at $T=4 K$. The large observed value $\epsilon=1,500$ provides further support for the theoretical prediction of electronic ferroelectricity. An interesting test would be the existence of the ferroelectric resonance in this compound. For the parameter values of Fig. 5, the ferroelectric resonance frequency is $\omega_{0}=0.021 \sqrt{E_{z}} \mathrm{meV}$, where $E_{z}$ is in $\mathrm{V} / \mathrm{cm}$. This estimate from Eq. (32) was checked by the numerical solution of $D\left(\omega_{0}\right)=0$ and was found to be in agreement to better than $0.5 \%$ for fields up to $10^{3} \mathrm{~V} / \mathrm{cm}$. For a reasonably strong applied electric field, the ferroelectric resonance lies easily in the range of frequency measured in Ref. [14] at zero static electric field since the lowest frequency measured there was $1 \mathrm{meV}$.

Ref. [24] reports measurements of the dielectric response of mixed-valent $\mathrm{Sm}_{3} \mathrm{Se}_{4}$ and $\mathrm{Sm}_{2} \mathrm{Se}_{3}$ from $20 \mathrm{~Hz}$ up to 1 GHz. The huge observed values of the static dielectric constant $\left(\epsilon=30,000\right.$ for $\mathrm{Sm}_{3} \mathrm{Se}_{4}$ and $\epsilon=4,000$ for $\left.\mathrm{Sm}_{2} \mathrm{Se}_{3}\right)$ are consistent with electronic ferroelectricity. Given the value $2 \Delta=140 \mathrm{meV}$ for the energy gap in $\mathrm{Sm}_{3} \mathrm{Se}_{4}$, we predict a resonance frequency between $0.1 \mathrm{meV}(10 \mathrm{GHz})$ and $10 \mathrm{meV}(1 \mathrm{THz})$.

Ref. 25] reports transport measurements on mixed-valent $\mathrm{TmSe}_{0.45} \mathrm{Te}_{0.55}$ that show evidence for a condensation of free carriers into an excitonic insulator ground state. Due to the indirect nature of the energy gap, this material might not be suitable for the optical tests of coherence proposed here.

\section{DISCUSSION OF OTHER GROUND STATES}

We now discuss the possibility of electronic ferroelectricity, ferroelectric resonance, and second-harmonic generation for solutions of the FK model other than the SCMF solution. There are two types of solutions we consider here: (1) solutions with a classical $f$-electron distribution, and (2) electronic-polaron solutions. The question we ask is: does the theoretical ground state have a built-in coherence between $d$-electrons and $f$-holes? This coherence is necessary for electronic ferroelectricity and the concomitant optical signatures to occur. The answer is, for the two types of solutions: (1) no, and (2) yes. A brief motivation for each answer is given below.

In Refs. [3, th the $f$-occupation $f_{i}^{\dagger} f_{i}$ is replaced by a classical variable $W_{i}$, where $W_{i}=1$ when site $i$ is occupied by an $f$-electron and $W_{i}=0$ when it is not. With this replacement, the FK model becomes a tight-binding model with an on-site potential that can assume two different values $U$ or 0 . For a given configuration of $f$-electrons the ground state energy is found by filling the lowest $d$-electron levels. The ground configuration is the configuration with the lowest ground state energy. The question we ask is: what is the value of $\Delta=\frac{U}{N} \sum_{i=1}^{N}\left\langle\psi\left|f_{i}^{\dagger} d_{i}\right| \psi\right\rangle$ ? Since each site is either occupied $\left(f_{i}^{\dagger} f_{i}=1\right)$ or empty $\left(f_{i}^{\dagger} f_{i}=0\right)$, every term in the sum vanishes, and $\Delta=0$ no matter what configuration of $f$-electrons we choose. 
However, we argue that there are degenerate states in the $f$-occupation representation, linear combinations of which are ground states with finite polarization. It is straightforward to demonstrate this property for the exact solutions of a ring of four sites. We construct a ring of four sites with $d$ - and $f$-states on alternate sites. The Hamiltonian, including all the FK terms, has inversion symmetry with respect to the $d$ - and $f$-sites. There are three eigenstates which are even under inversion with respect to a $d$-site, and three which are odd. The parameters are varied so that a degeneracy occurs between two states of odd parity and one of even parity. The ground state in the limit of a vanishing electric field is a mixture of even and odd states with a finite polarization.

In Ref. $[6]$ an electronic-polaron solution of the FK model is presented that may explain the anomalous properties of heavy-electron materials such as $\mathrm{UPt}_{3}, \mathrm{UBe}_{13}$, and $\mathrm{CeCu}_{2} \mathrm{Si}_{2}$. It is proposed that the Coulomb interaction causes the $f$-electrons in these materials to propagate like polarons, with a screening cloud made up of $d$-electrons. The model is shown to account for a large number of thermodynamic and transport properties of heavy-electron materials.

Here we show that the electronic polaron has a nonzero built-in coherence

$$
\Delta=\frac{U}{N} \sum_{\mathbf{k}} G_{d f}\left(\mathbf{k}, \tau=0^{-}\right)
$$

where $G_{d f}(\mathbf{k}, \tau)=-\left\langle T_{\tau} d_{\mathbf{k}}(\tau) f_{\mathbf{k}}^{\dagger}(0)\right\rangle$ is a mixed Green's function with Fourier transform $G_{d f}\left(\mathbf{k}, i \omega_{n}\right)=$ $-V G_{f f}\left(\mathbf{k}, i \omega_{n}\right) G_{d d}\left(\mathbf{k}, i \omega_{n}\right)$, where $G_{d d}$ and $G_{f f}$ are the $d$ - and $f$-electron propagators, and $\omega_{n}$ are the Matsubara frequencies. Expressing the Green's functions in the Lehmann representation, and summing over the Matsubara frequencies, we find (at $T=0$ )

$$
\Delta=V U A \pi \csc (\pi \alpha) \int_{\mu_{0}}^{\infty} d \omega \omega^{-\alpha} N_{+}(\omega)
$$

Here $A$ and $\alpha$ are the forefactor and the singularity index of the $f$-electron spectral function (see Ref. [6]), $N_{+}(\omega)$ is the density of $d$-electron states in the upper branch, and $\mu_{0}$ is the Fermi level at $T=0$. Using Eq. (8) of Ref. [6] for $N_{+}(\omega)$ we obtain

$$
\Delta=\frac{U}{4 V}\left(\frac{\eta}{\mu_{0}}\right)^{2 \alpha} W
$$

where $W$ is the bandwidth and $\eta$ the upper-branch threshold. Taking the parameter values $\alpha=0.8, \mu_{0}=1.3 \eta$, and $U=20 \mathrm{~V}$ from Ref. [6] we get $\Delta=3.28 \mathrm{~W}$.

\section{SUMMARY AND CONCLUSION}

In this paper we have investigated the linear and nonlinear optical characteristics of the Falicov-Kimball model. The SCMF solution of the periodic model results in the Bose-Einstein condensation of $d-f$ excitons. We found that the pairing of $d$-states of even parity with $f$-states of odd parity breaks the inversion symmetry of the underlying crystal, leading to electronic ferroelectricity. The valence transition is accompanied by a divergence of the static dielectric constant at the critical value of the $f$-level energy. The existence of electronic ferroelectricity in a given mixed-valent compound predicates on the dominance of the $d-f$ Coulomb interaction over the hybridization.

We have calculated both the linear and the second-harmonic susceptibilities of a model mixed-valent system within the SCMF approximation. The absorption spectrum of the mixed-valent system, when placed in an additional static electric field, consists of a peak at the ferroelectric resonance frequency and a continuum above the energy gap. The ferroelectric resonance frequency is proportional to the square root of the effective bias field, which depends, in addition to the applied static field, on the sample shape, domain structure, and crystal anisotropy. The continuum absorption has a threshold singularity when the $f$-level lies within the conduction band. The second-harmonic susceptibility is directly proportional to the amount of built-in coherence $\Delta$. The final-state Coulomb interaction enhances the second-harmonic conversion efficiency at $\omega=\Delta$ and $\omega=2 \Delta$. As experimental tests of the electronic ferroelectricity in mixed-valent compounds we proposed measurements of the static dielectric constant, the microwave absorption spectrum, and the second-harmonic susceptibility. The measured far-infrared absorption spectrum of $\mathrm{SmB}_{6}$ was found to be consistent with the model calculation.

We have also discussed the possibility of electronic ferroelectricity, ferroelectric resonance, and second-harmonic generation for two other theoretical ground states of the Falicov-Kimball model. The electronic-polaron state does have a built-in coherence comparable to the SCMF solution. A ground state with $f$-occupation as a good quantum number has no built-in coherence between $d$-electrons and $f$-holes. Such a state on its own is not ferroelectric, does 
not exhibit ferroelectric resonance, and cannot sustain second-harmonic generation. We have argued that degenerate ground states in the $f$-occupation representation can lead to ferroelectric ground states. The explicit numerical demonstration of such ground states is left for the future.

\section{ACKNOWLEDGEMENTS}

We wish to thank M. B. Maple, S. H. Liu, D. P. Arovas, and S. R. Renn for helpful discussions. This work was supported in part by NSF Grant No. DMR 94-21966 and in part by the Deutsche Forschungsgemeinschaft (DFG).

\section{APPENDIX}

In this appendix we give the explicit expressions for the functions $A(\omega)$ and $B(\omega)$ defined in Eqs. (26) and (27). Replacing $\frac{1}{N} \sum_{\mathbf{k}}$ by $\int d \epsilon \rho(\epsilon)$, with $\rho(\epsilon)=\theta(W-|\epsilon|) /(2 W)$, we obtain

$$
\begin{aligned}
& A(\omega)=\frac{U}{2 W} \int_{-W-E_{f}}^{W-E_{f}} d \epsilon \frac{1}{\sqrt{\epsilon^{2}+M^{2}}\left(\omega^{2}-\epsilon^{2}-M^{2}\right)} \\
& B(\omega)=\frac{U}{2 W} \int_{-W-E_{f}}^{W-E_{f}} d \epsilon \frac{\epsilon}{\sqrt{\epsilon^{2}+M^{2}}\left(\omega^{2}-\epsilon^{2}-M^{2}\right)} .
\end{aligned}
$$

The integrals can be performed by elementary methods. We find

$$
\begin{aligned}
A(\omega)= & \frac{U}{4 W^{2} \omega \sqrt{\omega^{2}-M^{2}}}\left[\ln \left(\frac{\sqrt{\left(E_{f}+W\right)^{2}+M^{2}} \sqrt{\omega^{2}-M^{2}}+\left(E_{f}+W\right) \omega}{\sqrt{\left(E_{f}+W\right)^{2}+M^{2}} \sqrt{\omega^{2}-M^{2}}-\left(E_{f}+W\right) \omega}\right)\right. \\
& \left.-\ln \left(\frac{\sqrt{\left(E_{f}-W\right)^{2}+M^{2}} \sqrt{\omega^{2}-M^{2}}+\left(E_{f}-W\right) \omega}{\sqrt{\left(E_{f}-W\right)^{2}+M^{2}} \sqrt{\omega^{2}-M^{2}}-\left(E_{f}-W\right) \omega}\right)\right]
\end{aligned}
$$

and

$$
B(\omega)=\frac{U}{4 W^{2} \omega}\left[\ln \left(\frac{\sqrt{\left(E_{f}+W\right)^{2}+M^{2}}+\omega}{\sqrt{\left(E_{f}+W\right)^{2}+M^{2}}-\omega}\right)-\ln \left(\frac{\sqrt{\left(E_{f}-W\right)^{2}+M^{2}}+\omega}{\sqrt{\left(E_{f}-W\right)^{2}+M^{2}}-\omega}\right)\right] .
$$

The frequency $\omega$ is understood to have a positive imaginary part $\delta$. The function $A(\omega)$ is even in $E_{f}$, while $B(\omega)$ is odd in $E_{f}$. This means $B(\omega)=0$ at half-filling.

The static susceptibility given by Eq. (10) involves the functions $A(\omega)$ and $B(\omega)$ evaluated at $\omega=0$. Taking the limit $\omega \rightarrow 0$ in Eqs. (50) and (51) we obtain

$$
\begin{aligned}
& A(0)=\frac{U}{2 W^{2} M}\left(\frac{W+E_{f}}{\sqrt{\left(W+E_{f}\right)^{2}+M^{2}}}-\frac{W-E_{f}}{\sqrt{\left(W-E_{f}\right)^{2}+M^{2}}}\right), \\
& B(0)=\frac{U}{2 W^{2}}\left(\frac{1}{\sqrt{\left(W-E_{f}\right)^{2}+M^{2}}}-\frac{1}{\sqrt{\left(W+E_{f}\right)^{2}+M^{2}}}\right) .
\end{aligned}
$$

FIG. 1. $f$-level occupancy $n_{f}$ and gap parameter $\Delta$ of the model system as a function of the $f$-level energy $E_{f}$. The Coulomb repulsion is $U=3.0 \mathrm{~W}$.

FIG. 2. Static dielectric constant $\epsilon_{z z}$ of the model system as a function of the $f$-level energy $E_{f}$. The inset shows the built-in polarization as a function of $E_{f}$. The Coulomb repulsion is $U=15 \mathrm{meV}$ and the electric-dipole matrix element is $\mu_{z}=3.710^{-29}$ $\mathrm{Cm}$. The parameter values were obtained by fitting the absorption spectrum of the model system to experimental data on $\mathrm{SmB}_{6}$ (see Fig. 5). 
FIG. 3. Absorption spectrum $\operatorname{Im} \chi_{z z}^{(1)}(\omega)$ of the model system as a function of the photon energy $\omega$, for various values of the $f$-level energy $E_{f}$. The Coulomb repulsion is $U=3.0 \mathrm{~W}$. The solid lines show the continuum absorption spectrum in zero bias field. The dash-dotted line shows both the ferroelectric resonance peak and the continuum absorption spectrum in a bias field of $E_{z}=0.01 \mu_{z} / W$.

FIG. 4. Amplitude $\left|\chi_{z z z}^{(2)}(2 \omega, \omega, \omega)\right|$ of the second-harmonic susceptibility as a function of the photon energy $\omega$, for various values of the $f$-level energy $E_{f}$. The dash-dotted line shows the phase of $\chi_{z z z}^{(2)}(2 \omega, \omega, \omega)$ for $E_{f}=-1.0 W$. The Coulomb repulsion is $U=3.0 \mathrm{~W}$. The amplitude is given in units of $N \mu_{z}^{3} /\left(2 \Omega W^{2}\right)$. For the parameter values given for the solid line in Fig. 5, $N \mu_{z}^{3} /\left(2 \Omega W^{2}\right)=82 \mathrm{nmV}^{-1}$.

FIG. 5. Comparison of the mean-field (solid line) and single-quasiparticle (dash-dotted line) results for the infrared absorption spectrum of $\mathrm{SmB}_{6}$ to experimental data taken from Ref. [14] (diamonds). The $f$-level energy is $E_{f}=0$, the $d$-bandwidth is $W=40 \mathrm{meV}$, and the Coulomb repulsion is $U=15 \mathrm{meV}$. The electric-dipole matrix element is $\mu_{z}=3.710^{-29} \mathrm{Cm}$ for the solid line, and $\mu_{z}=4.210^{-29} \mathrm{Cm}$ for the dash-dotted line.

[1] C. Kittel, Introduction to Solid State Physics (John Wiley, New York, 1986), Ch. 13.

[2] L. M. Falicov and J. C. Kimball, Phys. Rev. Lett. 22, 997 (1969); R. Ramirez, L. M. Falicov, and J. C. Kimball, Phys. Rev. B 2, 3383 (1970).

[3] J. K. Freericks and L. M. Falicov, Phys. Rev. B 41, 2163 (1990).

[4] P. Farkašovský, Phys. Rev. B 51, 1507 (1995).

[5] H. J. Leder, Solid State Commun. 27, 579 (1978).

[6] S. H. Liu, Phys. Rev. B 37, 3542 (1988);

[7] A brief account of some of the results of this work is given in T. Portengen, Th. Östreich, and L. J. Sham, Phys. Rev. Lett. 76, 3384 (1996).

[8] K. Leo et al., Phys. Rev. Lett. 65, 1340 (1990).

[9] S. Weiss et al., Phys. Rev. Lett. 69, 2685 (1992).

[10] D. S. Kim et al., Phys. Rev. Lett. 69, 2725 (1992).

[11] H. Fröhlich, Proc. R. Soc. London, Ser. A, 223, 296 (1954).

[12] S. Schmitt-Rink, D. S. Chemla, and H. Haug, Phys. Rev. B 37, 941 (1988).

[13] E. M. Fatuzzo and W. J. Merz, Ferroelectricity (North-Holland, Amsterdam, 1967).

[14] P. Wachter and G. Travaglini, J. Magn. Magn. Mat. 47-48, 423 (1985).

[15] S. von Molnar et al., in Valence Instabilities, edited by P. Wachter and H. Boppart (North-Holland, Amsterdam, 1982).

[16] B. Batlogg, P. H. Schmidt, and J. M. Rowell, in Valence Fluctuations in Solids, edited by L. M. Falicov, W. Hanke, and M. B. Maple (North-Holland, Amsterdam, 1981).

[17] G. Güntherodt, W. A. Thompson, F. Holtzberg, and Z. Fisk, in Valence Instabilities, edited by P. Wachter and H. Boppart (North-Holland, Amsterdam, 1982).

[18] G. D. Mahan, Many-Particle Physics, (Plenum, New York, 1990), p. 816.

[19] M. Tinkham, Introduction to Superconductivity, (McGraw-Hill, New York, 1975).

[20] J. R. Schrieffer, Theory of Superconductivity (Addison-Wesley, Redwood City, California, 1988), Sec. 3-5.

[21] S. V. Vonsovskii, Ferromagnetic resonance (Pergamon, Oxford, 1966).

[22] J. Zubia et al., Liquid Crystals 10, 849 (1991); S. U. Vallerien et al., Phys. Rev. A 42, 2482 (1990).

[23] M. Sparks, Ferromagnetic-relaxation theory (McGraw-Hill, New York, 1964).

[24] H. Goto et al., J. Phys. Jpn. 62, 1365 (1993).

[25] B. Bucher, P. Steiner, and P. Wachter, Phys. Rev. Lett. 67, 2717 (1991). 
PORTENGEN et al. PRB THEORY OF ELECTRONIC FERROELECTRICITY FIG. 1

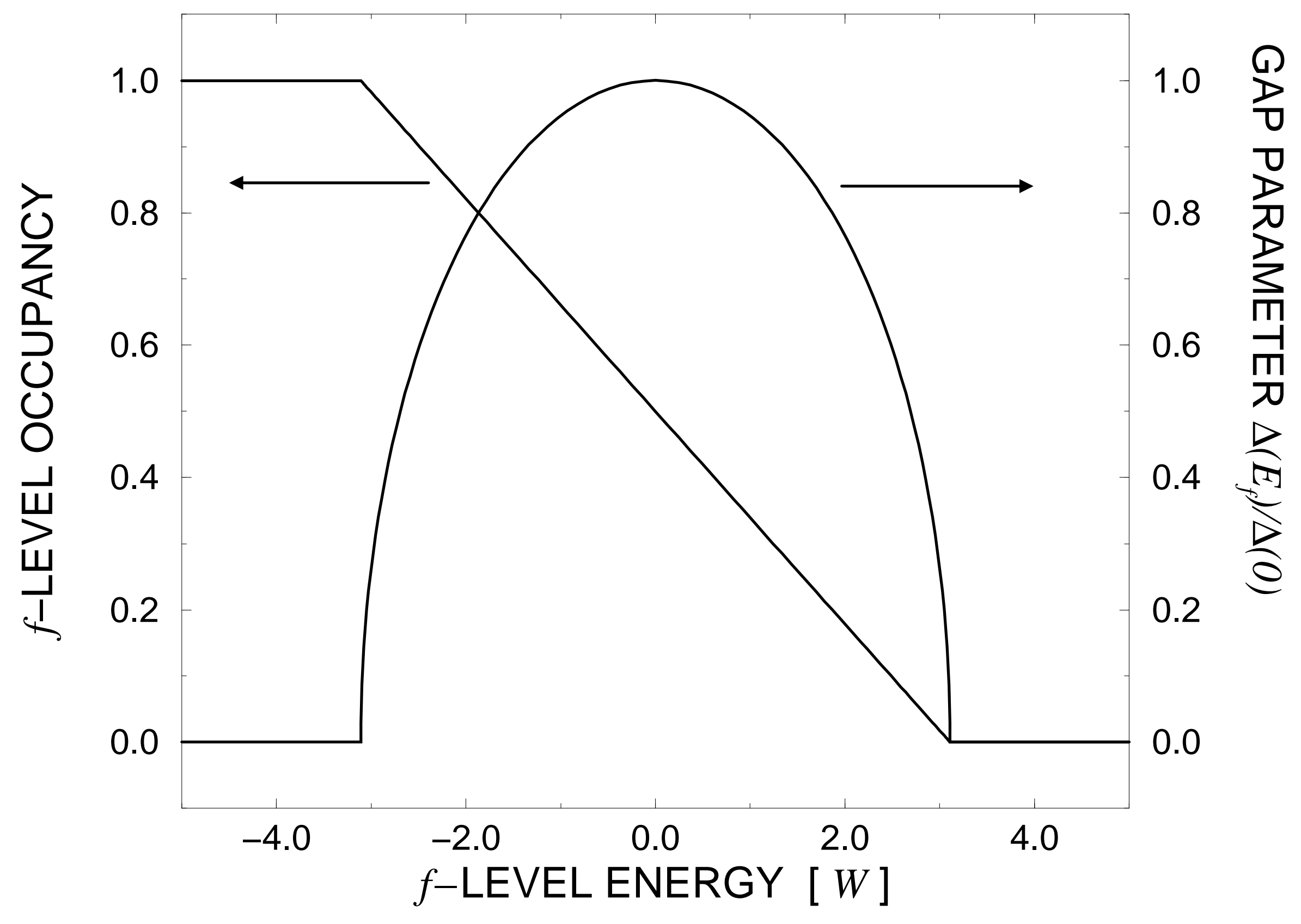




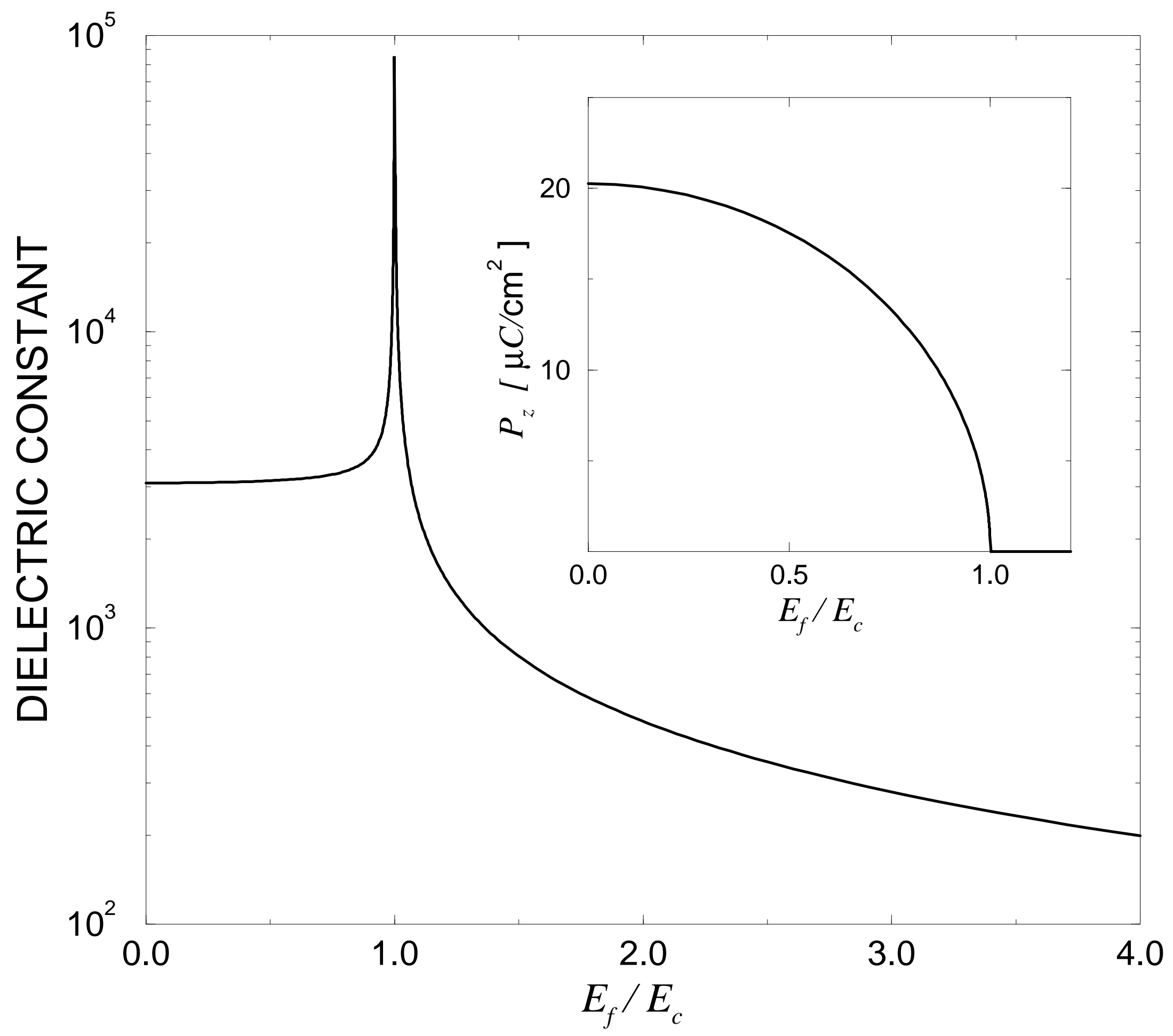

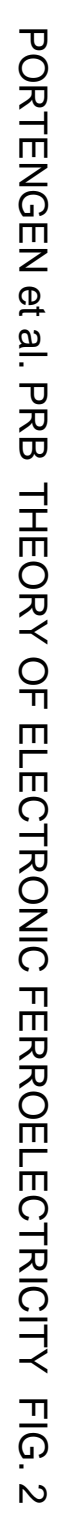




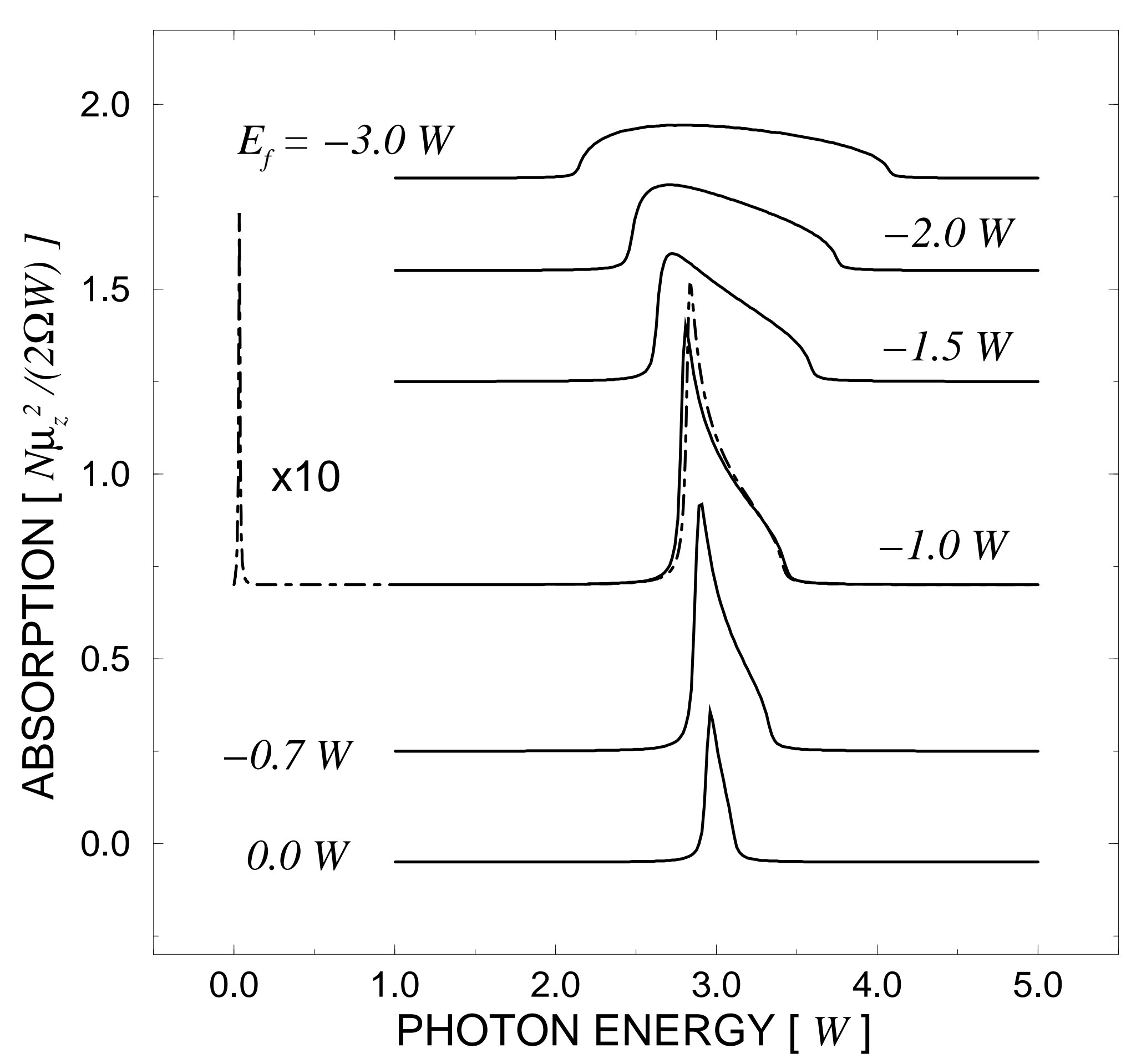

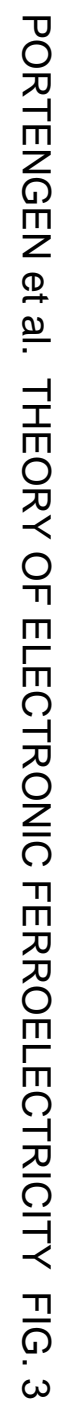




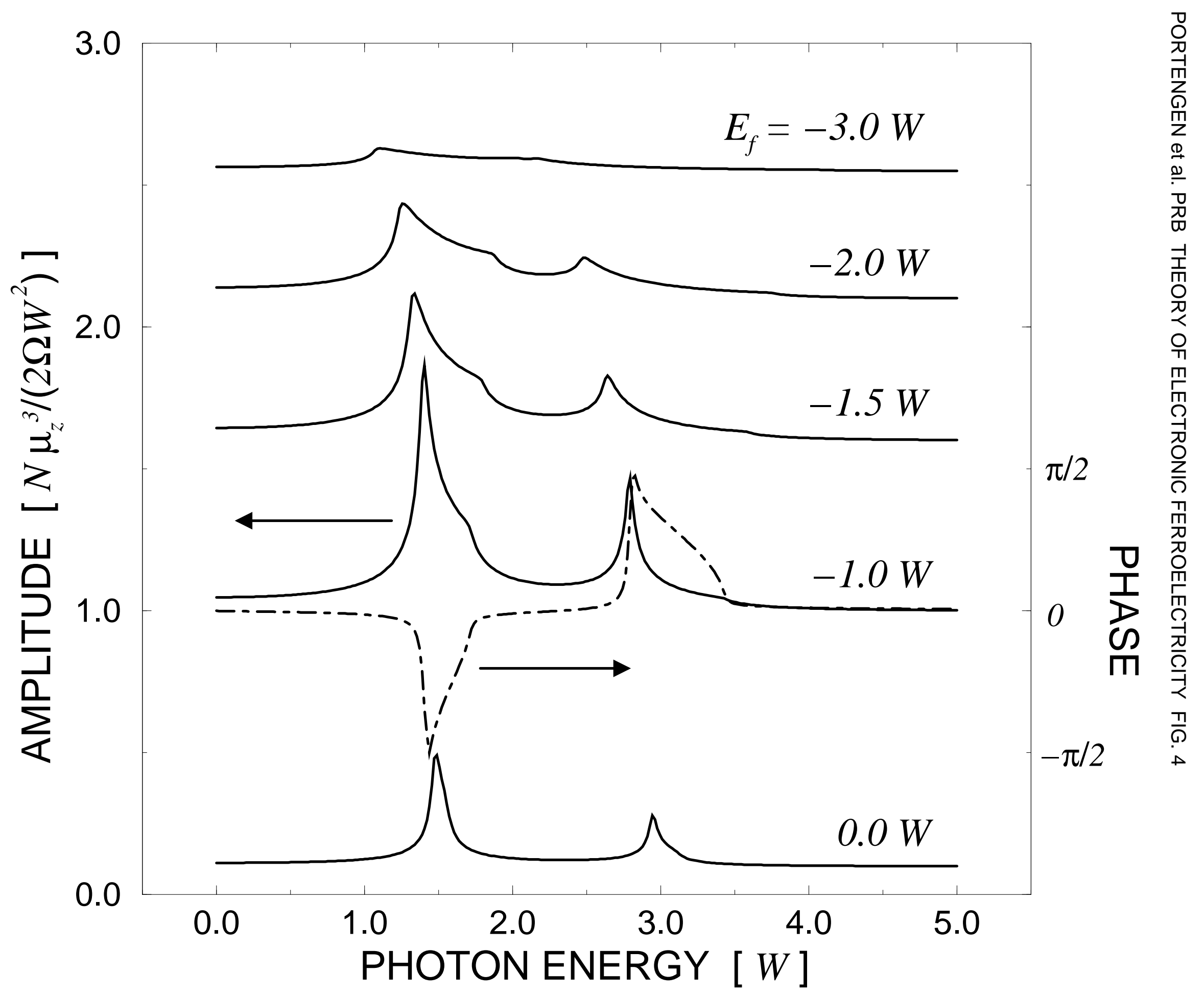




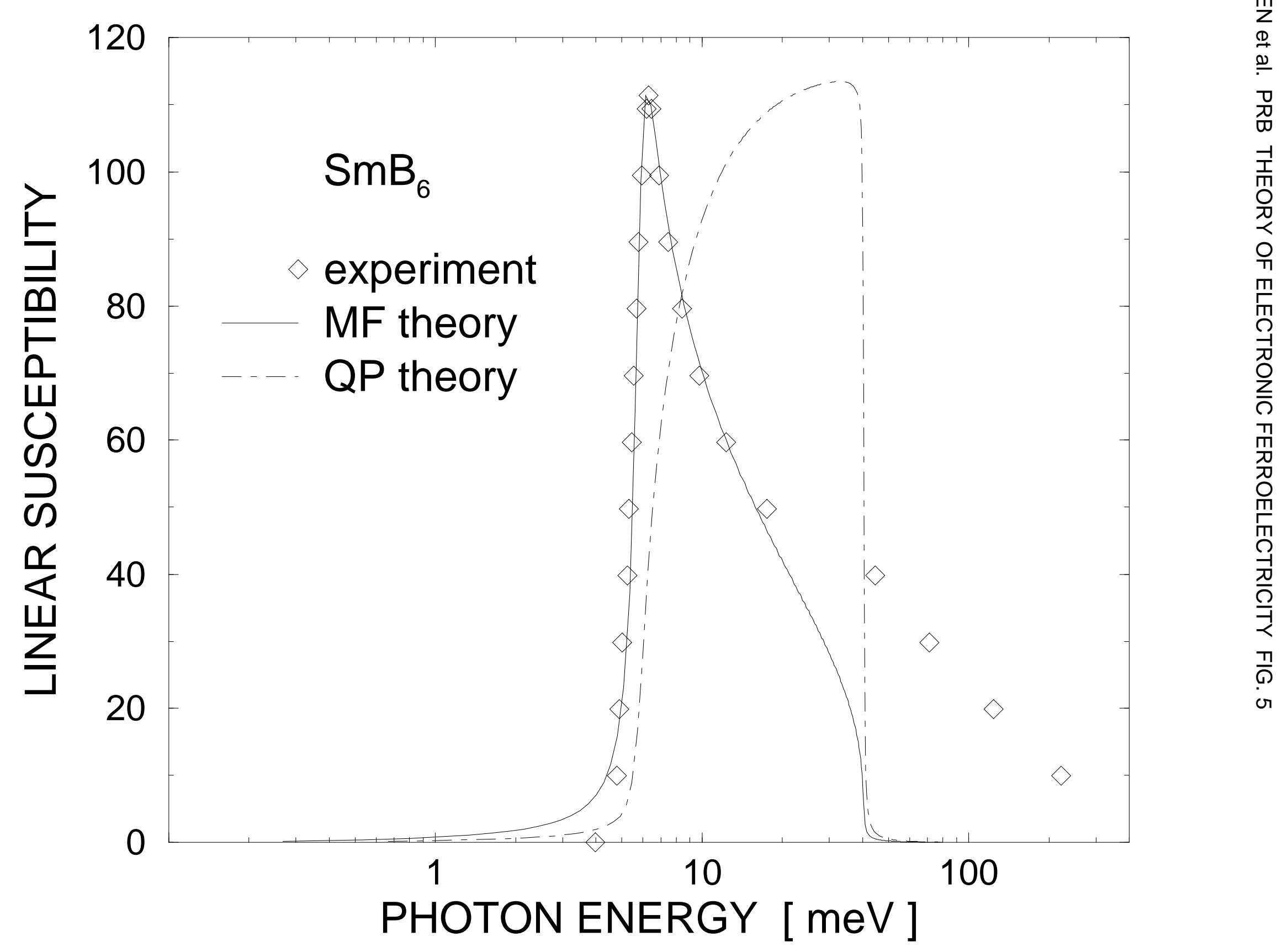

